\title{
Patrones biogeográficos de los helechos de las Sierras de Córdoba (Argentina) y sus implicancias en la conservación
}

\section{Biogeographical patterns of the ferns from Cordoba Hills (Argentina) and their implications in conservation}

\author{
Marcelo D. Arana ${ }^{1 *}$, Mónica Ponce ${ }^{2}$, Juan J. Morrone ${ }^{3}, \&$ Antonia J. Oggero ${ }^{1}$ \\ ${ }^{1}$ Orientación Plantas Vasculares, Departamento de Ciencias Naturales, Facultad de Ciencias Exactas, Físico-Químicas y \\ Naturales, Universidad Nacional de Río Cuarto, Ruta 36 km 601, X5804ZAB Río Cuarto, Córdoba, Argentina. \\ ${ }^{2}$ Instituto de Botánica Darwinion, Academia Nacional de Ciencias Exactas, Físicas y Naturales y CONICET, casilla de correo \\ 22, 1624 San Isidro, Argentina. \\ ${ }^{3}$ Museo de Zoología "Alfonso L. Herrera", Departamento de Biología Evolutiva, Facultad de Ciencias, Universidad Nacional \\ Autónoma de México (UNAM), Apdo. postal 70-399, 04510 México, D.F., México. \\ *marana@exa.unrc.edu.ar
}

\begin{abstract}
RESUMEN
Los atlas biogeográficos son la síntesis de patrones de distribución representada por trazos individuales, generalizados, nodos, áreas de endemismo y cladogramas de áreas, y a partir de ellos es posible establecer áreas prioritarias a conservar. Los helechos son indicadores de calidad de los hábitats y su falta de relaciones coevolutivas con vectores bióticos, así como su monofilia y estabilidad morfológica los hacen apropiados para establecer patrones biogeográficos. Nuestro objetivo fue aplicar análisis de trazos y parsimonia de endemismos a la flora de helechos de las Sierras de Córdoba (77 taxones) para contribuir a la elaboración del Atlas Biogeográfico centro-argentino. El patrón distribucional encontrado muestra las estrechas relaciones de estos helechos con las floras de las Sierras Subandinas; los Andes del Norte, los sistemas serranos de Buenos Aires y el sur de Brasil, correspondiéndose con el Arco Peripampásico Serrano. La protección de un espacio geográfico rico y complejo, con taxones endémicos y de distribución más amplia, garantiza preservar las condiciones en las que los taxones se desenvuelven. En este sentido, las sierras de Córdoba son un área prioritaria para conservar, porque constituyen un nodo o área biogeográficamente compleja, con una riqueza apreciable en especies de helechos y orígenes históricos, siendo un centro de convergencia biótica.
\end{abstract}

Palabras clave: Helechos, distribución, Chaco serrano, panbiogeografía, análisis de parsimonia de endemismos.

\begin{abstract}
Biogeographic atlases are the synthesis of distributional patterns represented by individual and generalized tracks, nodes, areas of endemism and area cladograms. They may help propose areas for conservation. Ferns are indicators of the quality of habitats and are especially useful for establishing biogeographic patterns due to their lack of coevolutionary relationships with biotic vectors, as well as their monophyly and morphological conservatism. Our aim was to apply a track analysis and a parsimony analysis of endemicity to the fern flora of the Córdoba Hills ( 77 taxa) in order to contribute to the Biogeographic Atlas of central Argentina. The established distributional pattern shows the closer relationships of the fern flora of central Argentina with the Subandean Hills, Peruvian and Ecuadorian Andes, Buenos Aires Hills and southern Brazil, corresponding to the "Serranean Peripampasic Arch". The protection of a rich and complex geographic area, with both endemic and widespread taxa, guarantees the preservation of conditions where the taxa develop. In this way, Cordoba Hills are a priority area for conservation, since they constitute a node or complex biogeographic area, with a remarkable fern richness of different historic origins, being a biotic convergence area.
\end{abstract}

KeYwords: Ferns, distribution, Chaco serrano, Panbiogeography, Parsimony Analysis of Endemicity. 


\section{INTRODUCCIÓN}

Los helechos se consideran un grupo monofilético, hermano de las plantas con semilla, que junto con las licofitas constituyen el conjunto de las plantas vasculares. Se caracterizan por sus hojas ricamente vascularizadas del tipo eufilos, excepcionalmente con hojas escamiformes y fusionadas, o con pequeños apéndices no vascularizados, con esporangios del tipo leptosporangios o eusporangios en general del lado abaxial de las hojas o de esporangióforos y protoxilema mesarco (Schneider et al. 2002, Smith et al. 2006). Se encuentran entre las plantas bioindicadoras (Lindenmayer et al. 2000), que son aquellas que poseen características morfológicas, ciclos de vida y requerimientos de microhábitats, que las hacen muy sensibles a las alteraciones ambientales (De la Sota 1973a, Page 1985, Moran 1995, Arcand \& Ranker 2008).

Comparados con las angiospermas, al igual que las licofitas, sus patrones de distribución están más relacionados con factores abióticos que en el caso de otras plantas que, en general, presentan relaciones coevolutivas a través de vectores bióticos (Barrington 1993, Moran 2008, Kessler 2010). Además, por su antigüedad, monofilia y estabilidad morfológica son especialmente indicados para establecer patrones biogeográficos (Arana et al. 2011). Por otra parte, son plantas consideradas extremadamente vulnerables a la extinción, debido a la fragmentación del hábitat y deforestación de bosques montanos tropicales y templados, donde los helechos presentan la mayor diversidad y se encuentran los nichos ecológicos que estas plantas requieren (Arcand \& Ranker 2008).

En la actualidad el alto grado de impacto antrópico sobre la biodiversidad está desencadenando altas tasas de extinción de especies que superan ampliamente las tasas naturales, en lo que se considera como el sexto evento de extinciones masivas en la historia de la vida en el planeta (Dirzo \& Raven 2003). Esta crisis de la biodiversidad, que se manifiesta en una creciente degradación y agotamiento de los ecosistemas, ha promovido en las últimas décadas un creciente interés científico, social, económico y ético por encontrar estrategias y criterios científicos sobre los que basar la conservación de la biodiversidad (Lobo 1996, Halffter 2000, Morrone 2000, Novacek 2008). Así, la principal actividad es acelerar el inventario de la biodiversidad y cuantificar la cantidad (expresada como riqueza de especies, rareza, endemicidad, diversidad filogenética) que alojan los ecosistemas. Para ello, el conocimiento de los taxones y las áreas de concentración de las especies son fundamentales para proponer una estrategia que permita el uso sustentable y conservación de la biodiversidad de una región determinada (Squeo et al. 1998, Morrone 2000, Mace 2004). Además este análisis resulta esencial para comprender el proceso evolutivo en tiempo y espacio, ya que la disposición espacial de los seres vivos es básicamente el resultado de la formación vicariante de los grupos taxonómicos involucrados (Craw et al. 1999). Este proceso vicariante, que incluye los procesos de movilidad (=dispersión) y vicarianza (Morrone 2009), nos permite entender a la biodiversidad desde una perspectiva histórica y generar información importante para la conservación (Morrone \& Espinosa 1998, Crisci et al. 2000, Morrone 2000). De acuerdo con la panbiogeografía, existen áreas denominadas nodos, que son el resultado de fragmentos geobióticos ancestrales diferentes que se superponen en espacio-tiempo (Morrone 2001a). Los nodos podrían representar hotspots o áreas con alta riqueza de especies, que además incluyen elementos taxonómicos de diverso origen, es decir, poseen diversidad de relaciones geográficas y filogenéticas (Morrone 2000, Aguilar-Aguilar \& Contreras 2001, Contreras-Medina et al. 2001, Escalante 2003, Arcand \& Ranker 2008, Arana et al. 2011), lo que los hace particularmente importantes para proponer áreas para conservación (Grehan 1993, Morrone \& Crisci 1995, Morrone \& Espinosa 1998, Craw et al. 1999, Heads 2004, Morrone 2004c, Contreras-Medina 2006).

Aunque hay una necesidad imperiosa de proteger las especies en peligro, los recursos dedicados a la conservación son limitados y deben estar enfocados, en parte, al reconocimiento y protección de las áreas vulnerables de alto valor biológico (Mittermeier 1988, Morrone 2000, Martínez-Aquino et al. 2007). La elaboración de atlas biogeográficos empleando métodos panbiogeográficos y cladísticos permitiría documentar de manera eficiente los patrones de la diversidad biológica, con miras a su conservación y uso sustentable (Morrone \& Espinosa 1998, Álvarez Mondragón \& Morrone 2004, Martinez-Aquino et al. 2007). Un atlas biogeográfico constituye la síntesis de los patrones de distribución de taxones de un país o área biogeográfica, representada por trazos, áreas de endemismo y cladogramas de áreas (Morrone 2000).

La provincia de Córdoba ocupa la región central de la Argentina y posee una gran diversidad de ecosistemas ocupando diferentes paisajes, como montañas, planicies y humedales. Presenta en general una típica vegetación xerófila chaqueña, que se empobrece florística y fisonómicamente de norte a sur, producto de procesos geológicos evolutivos que originaron una variedad de ambientes en su extensión (Luti et al. 1979). Por sus aptitudes climáticas y edáficas, ha sido fuertemente explotada por las actividades humanas. Particularmente, en las sierras de Córdoba los disturbios que suceden actualmente son el sobrepastoreo y los incendios en áreas de pastizales y arbustales; a los cuales se suma la tala y la deforestación en los bosques serranos (Bianco et al. 1987, Vischi et al. 2001, Verzino et al. 2005, Zak et al. 2008, Arana \& Oggero 2009, Oggero \& Arana 2012). Dado el alto nivel de degradación por los disturbios mencionados, se necesita un esfuerzo importante de protección (Bertonatti \& Corcuera 2000), para lo cual el conocimiento de su 
biodiversidad y relaciones biogeográficas es fundamental. Determinar la presencia de especies bioindicadoras resulta una forma de monitorear la calidad y estado del medio ambiente. La presencia de helechos y licofitas se corresponde con una mayor disponibilidad de agua y heterogeneidad de microambientes, por lo tanto sugiere una mayor biodiversidad.

Teniendo en cuenta lo expuesto, del mismo modo que con las licofitas (Arana et al. 2011), y sumado a que las Sierras de Córdoba constituyen el cuarto centro de diversidad de helechos y licofitas del Cono Sur (Arana et al. 2004, Arana \& Bianco 2009, 2011), nuestro objetivo es establecer las vinculaciones biogeográficas de la flora de helechos de Córdoba utilizando el análisis panbiogeográfico y el de parsimonia de endemismos (PAE) para contribuir a la elaboración del Atlas Biogeográfico de la región centro de Argentina y establecer prioridades de conservación.

\section{MATERIAL Y MÉTODOS}

Las sierras centrales de Argentina constituyen la entidad orográfica más oriental de las Sierras Pampeanas (Ramos 1999) y son un área de integración de elementos bióticos con distribuciones e historias biogeográficas variadas (Cabrera \& Willink 1973, Morrone 2001). Este sector serrano del centro de Argentina (Fig. 1) se encuentra inmerso en la provincia biogeográfica del Chaco, dentro de la subregión Chaqueña, que ocupa el norte y centro de la Argentina, sur de Bolivia, oeste y centro del Paraguay, y centro y noroeste del Brasil (Morrone 2000, 2001b, 2006), formando parte de la región Neotropical (Morrone 2006). La vegetación de estos ambientes serranos consiste en bosques caducifolios xéricos, cuyas especies arbóreas dominantes son Lithraea molleoides (Vell.) Engl., "molle", Zanthoxylum coco Gillies ex Hook.f. \& Arn., "coco" y Vachellia caven (Molina) Seigler \& Ebinger, "espinillo" (Oggero \& Arana 2012). Las laderas con exposición norte y oeste son más secas y cálidas y entre su vegetación se incluye Schinopsis lorentzii (Griseb.) Engl., “orcoquebracho”. Como especies leñosas acompañantes se pueden encontrar Celtis ehrembergiana (Klotzsch) Liebm., "tala" y Colletia spinosissima J.F. Gmel., "tola", junto a estratos de poáceas, cactáceas y bromeliáceas.

Las unidades de estudio fueron los 77 taxones específicos e infraespecíficos que habitan las Sierras de Córdoba (los adventicios no se tomaron en cuenta). En el Apéndice 1 se brinda la lista de los taxones estudiados que se presenta de acuerdo al sistema de clasificación propuesto por Chase \& Reveal (2009) y Christenhusz et al. (2011), incluidas dentro de la Clase Embryopsida (Pirani \& Prado 2012).

Para establecer la distribución de las 77 entidades de helechos presentes en las Sierras de Córdoba, se reunió la información proveniente de antecedentes bibliográficos
(Hieronymus 1896, Hicken 1908, 1919, Pastore 1936, Capurro 1969, De la Sota 1967, 1973, 1977, Tryon \& Tryon 1982, Rodríguez 1995, Ponce 1996, de la Sota et al. 1998, 2009, Arana et al. 2004, Ponce et al. 2002, Arana \& Bianco 2009, 2011, Arana 2012) y de la revisión de ejemplares provenientes del área bajo estudio y depositados en los herbarios BA, CORD, JUA, MCNS, MVFA, LIL, LP, RCV, RIOC, SI y SRFA (Thiers 2013); el material representativo estudiado se cita en el Apéndice 2. Además se consultaron las bases de datos del Instituto Darwinion (www.darwin.edu.ar/Proyectos/ FloraArgentina/FA.asp), Missouri Botanical Garden (www. tropicos.org), Global Biodiversity Information Facility (www. gbif.org), Flora Argentina (http://www.floraargentina.edu. ar/publicaciones.asp) y de plantas endémicas de Argentina, PlanEAR (http://www.lista-planear.org).

Para establecer las afinidades de la biota de helechos de las Sierras de Córdoba con las otras áreas de América del Sur, se utilizaron la panbiogeografía y el análisis de parsimonia de endemismos (PAE), este último a dos niveles, las áreas de endemismo delimitadas por Crisci et al. (2001) ya que estas sierras forman parte del área de endemismo Sierras Pampeanas, y los centros de diversidad de helechos y licofitas de acuerdo con Ponce et al. (2002).

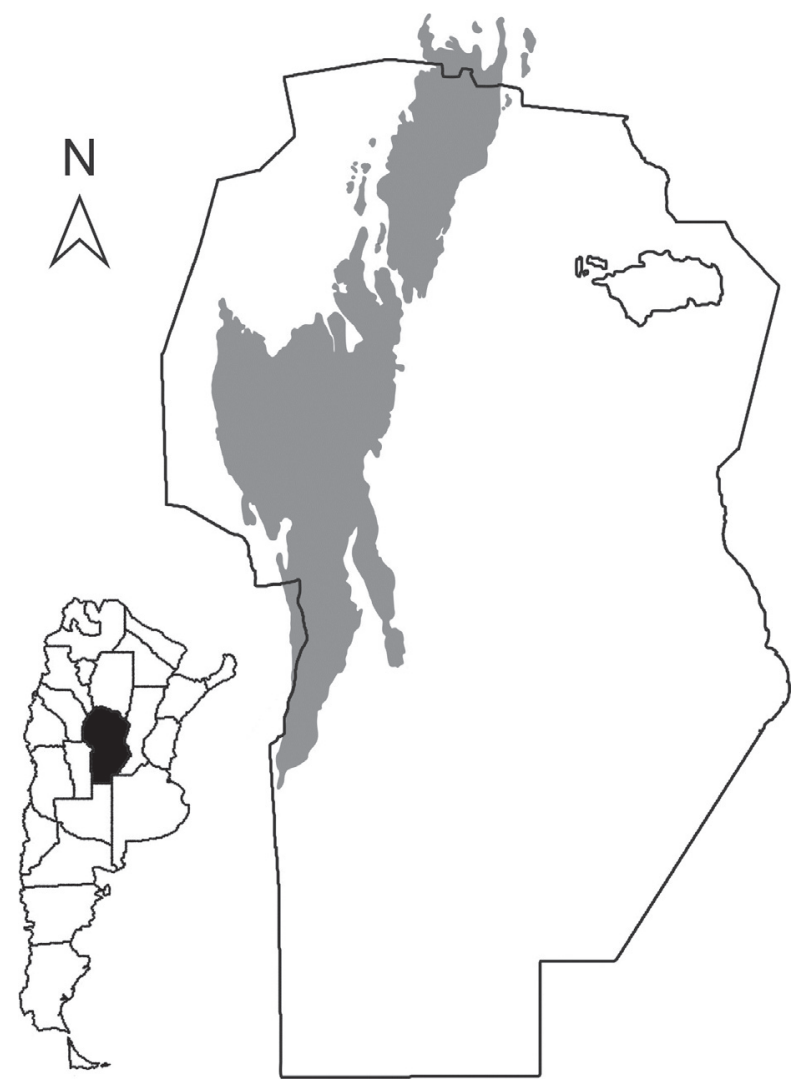

Figura 1. Área de estudio. La zona gris representa la ubicación y los límites de las sierras de Córdoba.

FIgURE 1. Study Area. The gray area shows the location and limits of the Cordoba hills. 
De acuerdo con la panbiogeografía, existe un único sistema geobiótico que evoluciona en el tiempo, en donde la diversidad biológica es un fenómeno espacio-temporal y existen líneas coincidentes de distribución de distintos taxones. Esto permite establecer homologías espaciales o biotas ancestrales y reconocer si las áreas poseen orígenes complejos, ya que los cambios tectónicos inducen cambios bióticos y los patrones de distribución son informativos acerca de la evolución biótica (Craw et al. 1999, Morrone 2009). Para llevar a cabo el análisis panbiogeográfico se graficaron los trazos individuales, y luego se establecieron los trazos generalizados y nodos. Los trazos generalizados representan patrones de distribución actuales de biotas ancestrales, las cuales fueron fragmentadas por eventos geológicos o tectónicos (Craw et al. 1999), y se obtuvieron mediante la superposición gráfica de los trazos individuales, donde se considera que dos o más trazos son compatibles solo si son iguales o uno se sitúa dentro del otro. Los nodos o centros de convergencia biótica se obtuvieron gráficamente donde dos o más trazos generalizados se superponen.

El análisis de parsimonia de endemismos (PAE) es una herramienta de la biogeografía histórica que permite descubrir los patrones naturales de distribución de los organismos, clasifica localidades, cuadrículas o áreas (análogas a taxones de la sistemática filogenética) de acuerdo con sus taxones compartidos (análogos a caracteres) mediante la solución más parsimoniosa (Crisci et al. 2000, 2001). Las áreas de endemismos con sus correspondientes acrónimos, son: Chile central (CHI), América Central y del Norte (CNA), Chaco (CHA), Mahuidas (MA), Andes del Norte (NA), Pampa (PAM), Patagonia (PAT), Sierras
Pampeanas (SPA), Sierras Subandinas (SA), Sur de Brasil (SB), Tandilia (TA), Uruguay (UR) y Ventania (VE). Los centros de diversidad de helechos en Argentina y Chile continental son los siguientes: Centro Subtropical del Nordeste (CSNE), Centro Subtropical del Noroeste (CSNO) y Centro Templado Sur (CTS).

Para el PAE se construyeron dos matrices (Apéndice 3), una de áreas de endemismos versus taxones presentes en Córdoba y otra de centros de diversidad versus los taxones que habitan Córdoba. En ambas, la presencia de una especie en un área determinada se codificó con 1 y su ausencia con 0 . El análisis se llevó a cabo con PAUP* versión 4.0 beta 2 (Swofford 1999), aplicando la opción "branch-and-bound", la cual calcula la solución exacta para el árbol con menor número de pasos (Goloboff 1998). Para llevar a cabo el proceso de análisis, los cladogramas fueron enraizados con un área hipotética (ANCES) codificada con 0.

\section{RESULTADOS}

\section{Panbiogeografía}

En los patrones distribucionales de la flora de helechos de la zona serrana de Córdoba obtenidos, se observa un trazo individual cosmopolita (Cystopteris fragilis) y un trazo generalizado Gondwánico, conectando América del Sur con África, Madagascar, India, Nueva Zelanda y Australia, al cual contribuyen las especies Adiantum thalictroides, Asplenium monanthes, Blechnum pennamarina, Botrychium australe subsp. australe, Cystopteris diaphana, Doryopteris concolor y Woodsia montevidensis (Fig. 2).

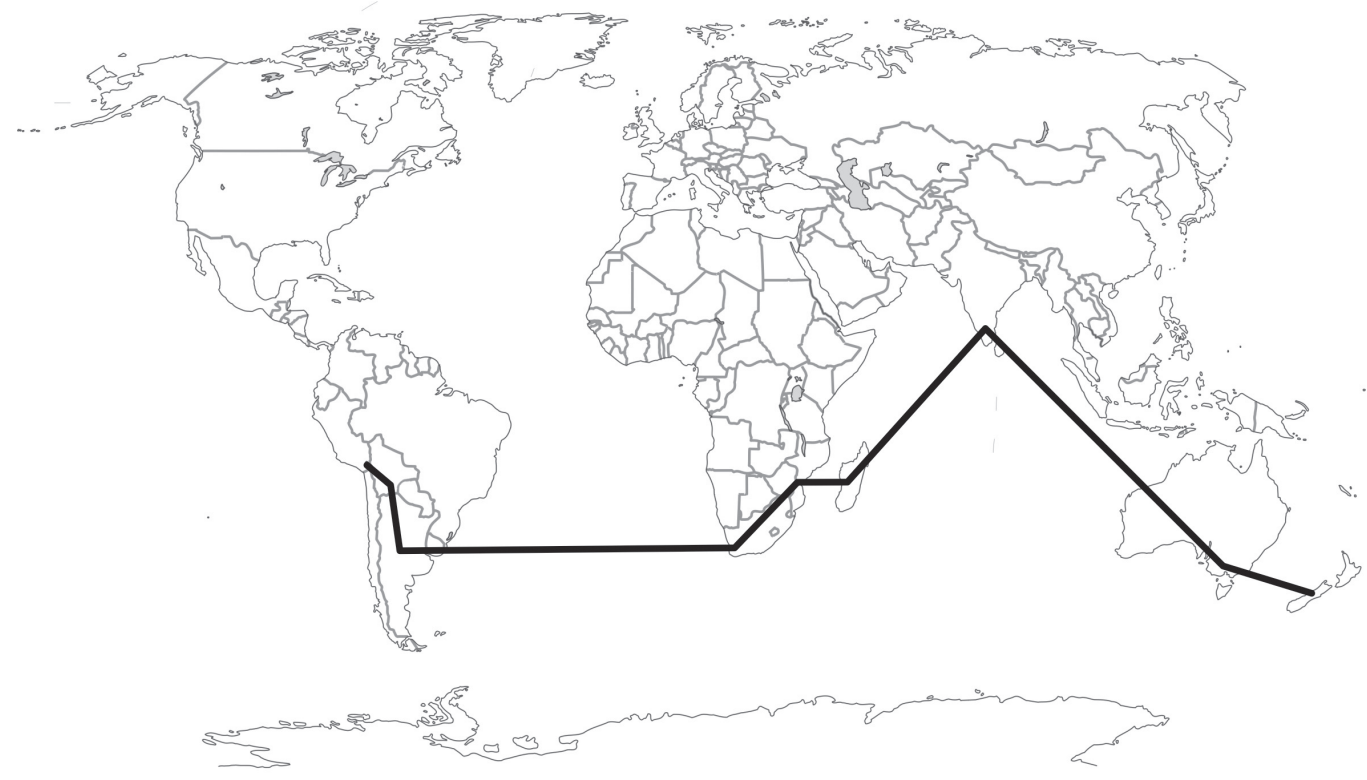

FiguRa 2. Trazo generalizado Gondwánico.

FIGURE 2. Gondwanic generalized track. 
El patrón distribucional principal en este análisis de superposición de trazos, es el determinado por 43 trazos de los 77 establecidos (trazo generalizado A en la Fig. 3, especies marcadas con * en el Apéndice 1), formando un trazo generalizado que conecta las sierras Pampeanas de Córdoba (Sierras Pampeanas sensu stricto o Sierras Pampeanas orientales) con las Sierras Subandinas y los Andes Bolivianos y Peruanos, llegando incluso hasta la Zona de Transición Mexicana al norte y los sistemas serranos de Buenos Aires al sur. Este último trazo generalizado está ejemplificado por los helechos Adiantum orbygnianum, Cheilanthes bonariensis, C. marginata, Melpomene peruviana, Notholaena sulphurea y Pityrogramma calomelanos var. austroamericana.

Sólo tres trazos individuales establecen conexión entre las Sierras de Córdoba y la región Andino-patagónica (Blechnum hastatum, B. mochaenum var. achalense y Elaphoglossum gayanum), formando el trazo generalizado C (Fig. 3).

La relación entre las sierras cordobesas con la región austral brasileña está dada por el trazo generalizado formado por los trazos individuales del complejo Anemia tomentosa y por los de Anogramma lorentzii, Blechnum australe subsp. auriculatum, Cassebeera triphylla, Microgramma vacciniifolia y Pleopeltis minima (trazo generalizado B, Fig. 3). La confluencia de los diferentes trazos generalizados generan un nodo en las Sierras de Córdoba (Fig. 3), lo

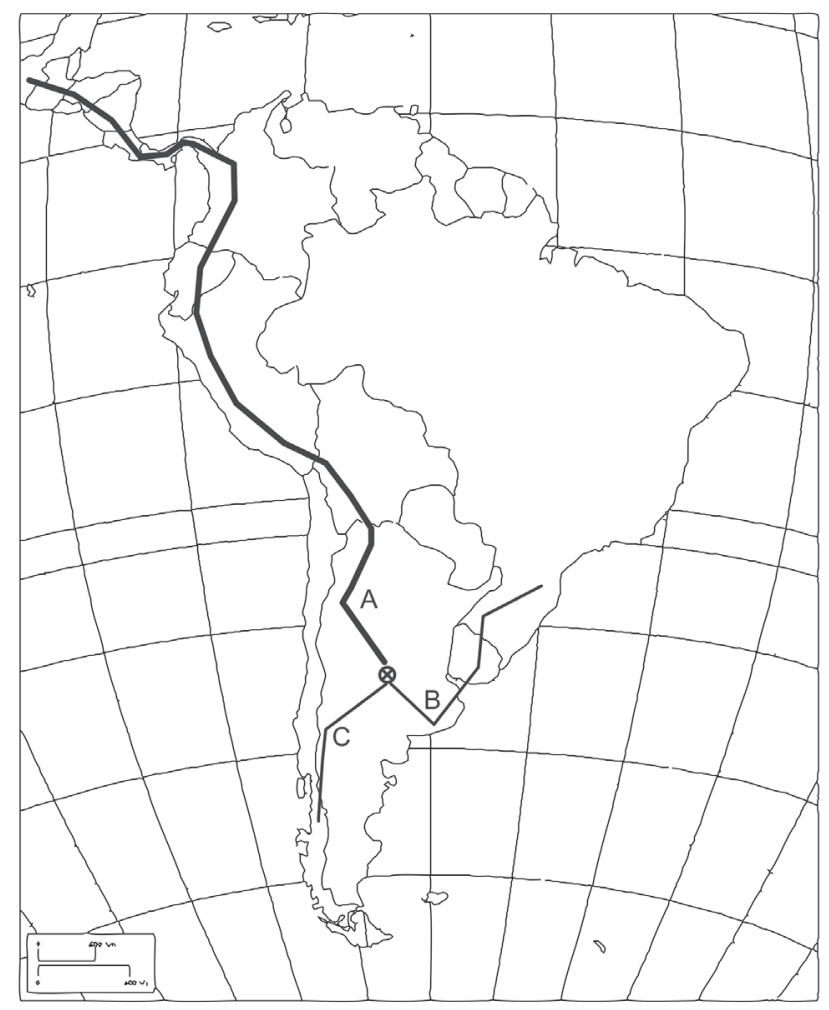

Figura 3. Trazos generalizados y nodo.

FIGURE. 3. Generalized tracks and node. que implica que a esta zona confluyen biotas de distintos orígenes e historias evolutivas.

Asimismo en las Sierras Pampeanas sensu lato existen taxones endémicos como Adiantum camptorachis, Anemia australis, Asplenium achalense, A. lilloanum, Blechnum squamipes, Hymenophyllum tunbrigense var. cordobense, Hypolepis poeppigii y Thelypteris achalensis, que conforman el trazo generalizado Sierras Pampeanas (Fig. 4); se podría incluir aquí a Pleopeltis pinnatifida y Serpocaulon gilliesii, que poseen una distribución amplia hasta las sierras australes de Buenos Aires. Este trazo generalizado está incluido en el trazo generalizado principal.

Cabe agregar que se encuentran dos especies exóticas creciendo espontáneamente en las sierras de Córdoba: Pteris vittata y Thelypteris dentata. La primera de ellas crece en ambientes urbanos y periurbanos sobre paredes de construcciones antiguas, mientras que la segunda crece en sitios húmedos y sombríos en los bordes de arroyos serranos.

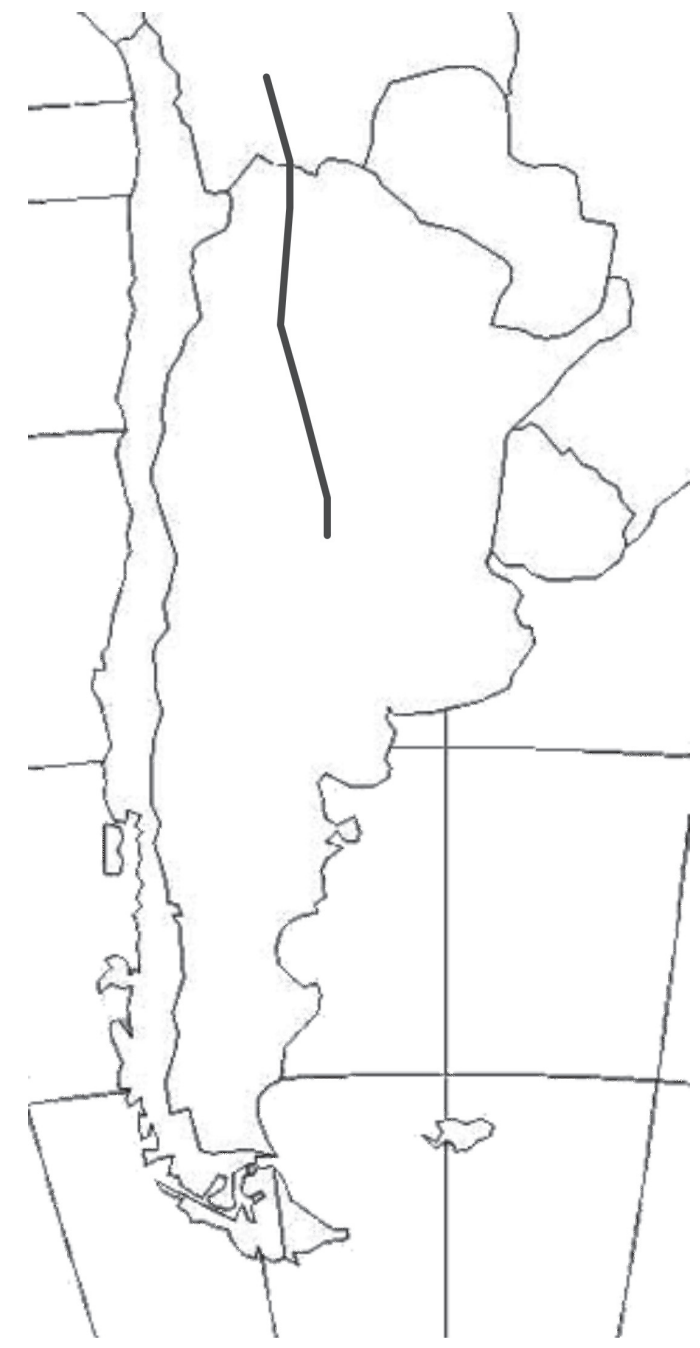

FIgura 4. Trazo generalizado Sierras Pampeanas.

Figure 4. Generalized track Pampean Hills. 
ANÁlisis DE PARSIMONIA DE ENDEMISMOS USANDO LAS ÁREAS DE ENDEMISMO

El cladograma de consenso estricto obtenido para el análisis utilizando las áreas de endemismo posee 173 pasos con un índice de consistencia (CI) de 0,4509 y un índice de retención (RI) de 0,6607 (Fig. 5). El cladograma representa áreas de endemismo anidadas, donde se observa una estrecha relación de las Sierras Pampeanas con las Sierras Subandinas (grupo 1, Fig. 5, soportado por las especies marcadas con 1 en el Apéndice 1), luego con los Andes del Norte (grupo 2, Fig. 5 , soportado por las especies marcadas con 2 en el Apéndice 1), seguido por el grupo Tandilia-Ventania (grupo 3, Fig. 5, soportado por las especies marcadas con 3 en el Apéndice 1). Luego se unen el Sur de Brasil (grupo 4, Fig. 5, soportado por

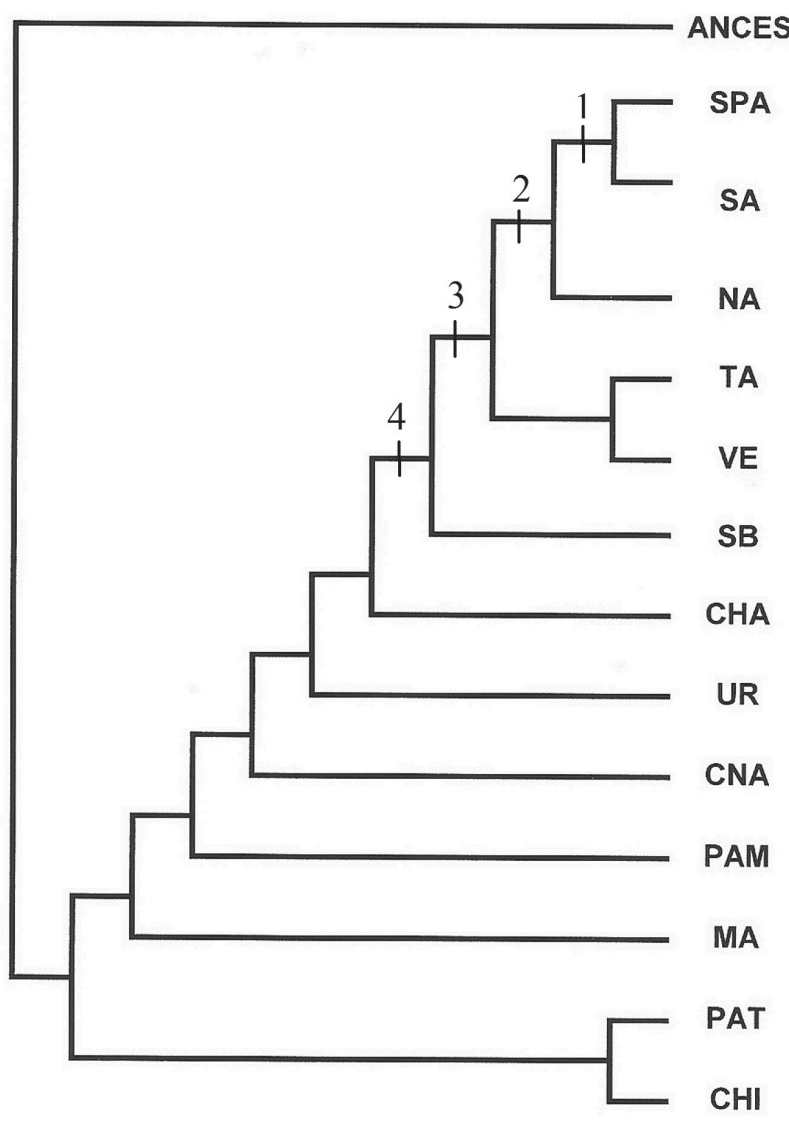

FIgURA 5. Cladograma de consenso estricto obtenido utilizando las áreas de endemismo. CHA: Chaco, CHI: Chile central, CNA: América Central y del Norte, MA: Mahuidas, NA: Andes del norte, PAM: Pampa, PAT: Patagonia, SA: Sierras Subandinas, SB: sur de Brasil, SPA: Sierras Pampeanas, TA: Tandilia, UR: Uruguay, VE: Ventania.

FIGURE 5. Strict consensus cladogram obtained using areas of endemism. CHA: Chaco, CHI: central Chile, CNA: Central and North América, MA: Mahuidas, NA: Northen Andes, PAM: Pampa, PAT: Patagonia, SA: Subandean Hills, SB: Southern Brasil, SPA: Pampean Hills, TA: Tandilia, UR: Uruguay, VE: Ventania. las especies marcadas con 4 en el Apéndice 1), seguido por el Chaco; al grupo formado anteriormente se le une Uruguay seguido de la Pampa. El grupo formado por Patagonia-Chile central no muestra relaciones resueltas con las otras áreas.

ANÁlisis DE PARSIMONIA DE ENDEMISMOS USANDO CENTROS DE DIVERSIDAD

El cladograma de consenso estricto obtenido para el análisis utilizando los centros de diversidad posee 90 pasos con un índice de consistencia (CI) de 0,8667 y un índice de retención (RI) de 0,8286 (Fig. 6). En este cladograma se observa una estrecha relación entre las Sierras de Córdoba y el centro subtropical del noroeste (grupo 5, Fig. 6, soportado por las especies marcadas con 5 en el Apéndice 1), luego ambas regiones se vinculan con el centro subtropical del nordeste (grupo 6, Fig. 6, soportado por las especies marcadas con 6 en el Apéndice 1), y no presentan relaciones resueltas con el centro templado del sur.

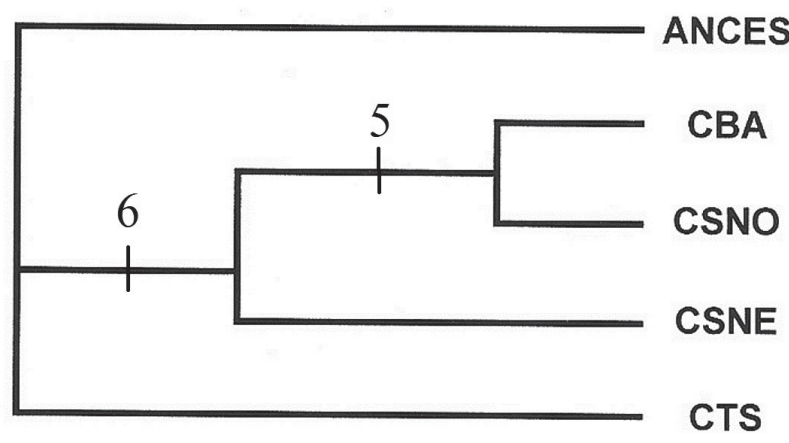

Figura 6. Cladograma de consenso estricto obtenido utilizando los centros de diversidad. CBA: Sierras de Córdoba, CSNO: centro subtropical del Noroeste, CSNE: centro subtropical del nordeste, CTS: centro templado del sur.

FIGURE 6. Strict consensus cladogram obtained using diversity centers. CBA: Córdoba Hills, CSNO: North-western subtropical center, CSNE: North-Eastern subtropical center, CTS: South template center.

\section{DISCUSIÓN Y CONCLUSIONES}

De acuerdo con la biogeografía evolutiva, la homología biogeográfica es uno de los principales patrones biogeográficos. Homología significa "equivalencia de partes", siendo el procedimiento empleado para establecer comparaciones taxonómicas válidas (De Pinna 1991). Partiendo de la analogía entre sistemática y biogeografía, podemos considerar las distribuciones de taxones individuales como los enunciados sobre la homología biogeográfica que comparamos (Morrone 2001a), reconociendo los mismos estadios que De Pinna (1991) 
propuso para la sistemática: la homología primaria (estadio de generación de hipótesis) y la secundaria (estadio de legitimación de hipótesis). De esta manera la homología biogeográfica primaria es una hipótesis intuitiva y deriva de la observación pura, por ejemplo, si la distribución de dos o más taxones coincide, se puede estimar que pertenecen a una biota ancestral, es decir que los diferentes taxones -aun teniendo medios de dispersión diferentes- se hallan integrados espacio-temporalmente en un mismo componente biótico (Morrone 2001). En este trabajo se utilizó el concepto de la panbiogeografía, que trata la homología biogeográfica primaria (Morrone 2001a, 2004), mientras que la biogeografía cladística trata la homología biogeográfica secundaria, corroborada a partir de la filogenia de los taxones (Crisci et al. 2000). Es posible integrarlas como etapas sucesivas de un mismo análisis (Morrone 2007), identificando los componentes bióticos y cenocrones: los primeros son los elementos bióticos sincrónicos que se observan en un tiempo determinado en el área en estudio, y los segundos son cada una de las biotas ancestrales que han dado origen a los componentes bióticos. Además se utilizó el análisis de parsimonia de endemismos (PAE), que permite descubrir los patrones naturales de distribución de los organismos pertenecientes a los componentes bióticos, al comparar distribuciones aplicando el análisis de parsimonia sobre la base de las especies presentes.

El trazo generalizado Gondwánico (Fig. 2) contribuye al patrón distribucional Gondwánico, ya demostrado para muchas especies actuales, por ejemplo Phlegmariurus saururus (Arana et al. 2011) como fósiles (Sanmartín \& Ronquist 2004), que conecta América del Sur con África, Madagascar, India, Nueva Zelanda y Australia. Este patrón de distribuciones disyuntas transoceánicas ha resultado de la ruptura secuencial del supercontinente Gondwana durante los últimos 165 millones de años, causando la división vicariante de su biota ancestral (Raven \& Axelrod 1972, Craw 1982, Swenson et al. 2001, Van Bocxlaer et al. 2006).

El principal patrón distribucional obtenido conecta los Andes del norte, las Sierras Pampeanas con las Sierras Subandinas, el grupo Tandilia-Ventania y el sur de Brasil (Fig. 3). Se obtuvo mediante los análisis panbiogeográficos y de parsimonia de endemismos (PAE), utilizando áreas de endemismo y los centros de diversidad y concuerda en forma general con las hipótesis de varios autores (Hicken 1919, Frenguelli 1950, De la Sota 1967, 1972, 1973, 1985, Acosta 1993, 2002). Los cladogramas resultantes representan áreas de endemismo anidadas $\mathrm{y}$, para el caso de los centros de diversidad, la relación entre los centros de diversidad, establecidos por Ponce et al. (2002), con la flora de helechos presente en las Sierras de Córdoba. Para ambos tratamientos, las dicotomías terminales (Sierras PampeanasSierras Subandinas y Sierras Pampeanas-Centro Noroeste) representan dos áreas entre las cuales ha ocurrido el más reciente intercambio de biotas (Morrone \& Crisci 1995).

Las relaciones de la biota de los Andes y Brasil, a través de las Sierras Pampeanas, Sierras de Buenos Aires y el Planalto y Sierras del sur de Brasil ya fueron postuladas por Hicken (1919). Del mismo modo, Frenguelli (1950) nombró como Arco Peripampásico Serrano al corredor constituido por las Sierras Pampeanas, Mahuidas y Tandilia. De la Sota (1967, 1972, 1973, 1985) en sus análisis de la flora de helechos y licofitas de Tandilia y Ventania, y el cono sur de América meridional, estableció que las montañas de Buenos Aires y Sierras Pampeanas constituyen estaciones intermediarias en la migración entre las floras Andino-Pampeana y las floras Australantártica y Austrobrasileña.

En este trabajo las relaciones entre las biotas analizadas muestran que las Sierras de Córdoba (incluidas clásicamente en el sistema de las Sierras Pampeanas) están estrechamente vinculadas con las áreas de endemismo de las Sierras Subandinas y Andes del Norte y con el centro de diversidad Centro Subtropical del Noroeste (área más o menos coincidente con las Sierras Subandinas), más que con las otras áreas analizadas; esto apoyaría la existencia de una flora ancestral que se fragmentó por eventos tectónicos o cambios climáticos. Además es posible inferir, a partir de los resultados obtenidos, que el intercambio florístico entre las Sierras de Córdoba y las Sierras Subandinas es particularmente intenso. Este patrón principal encontrado para los helechos es compartido por otros taxones, entre los que podemos citar los musgos de los géneros Rauiella Reimers y Thuidium Bruch \& Schimp. de la familia Thuidiaceae (Schiavone \& Suárez 2007), las licofitas (Arana et al. 2011), las especies de Junellia Moldenke, familia Verbenaceae (O' Leary et al. 2011) y las especies pertenecientes al subgénero Puyopsis (Baker) L.B. Smith (género Puya Molina) de la familia Bromeliaceae (Smith 1974). Entre los animales, este patrón es compartido por especies de escorpiones del género Orobothriurus Maury, familia Bothriuridae (Ochoa 2005); lepidópteros de los géneros Erinnyis Hübner, Euryglottis Boiduval, Manduca Hübner y Xylophanes Hübner, todos pertenecientes a la familia Sphingidae (Moré et al. 2005); anfibios como el grupo cinerea del género Pleurodema Tschudi, Leiuperidae (Cei 1980); reptiles de los géneros Liolaemus Wiegmann (Liolaemidae), Cercosaura Wagler (Gymnophtalmidae), y Stenocercus Duméril \& Bibron de la familia Tropiduridae (Cei 1986) y mamíferos, tanto fósiles como los xenartros de los géneros Chorohates Reig, Macrochorohates Scillato-Yané y Doellotatus Bordas (Prado et al. 1998); como actuales, por ejemplo Leopardus jacobitus de la familia Felidae (Wright 2004) y Tremarctos ornatus, único representante de la familia Ursidae en la región Neotropical (Del Moral Sachetti \& Lameda Caracaro 2011). Por otro lado, las Sierras Subandinas están estrechamente vinculadas con los Andes del Norte debido a que el CSNO comparte 159 de sus 182 especies de helechos con Bolivia (Ponce et al. 2002). 
Las conexiones florísticas entre las sierras de Buenos Aires y el sur de Brasil, un patrón ya demostrado para las Asteraceae (Crisci et al. 2001), también se encuentra en los helechos, como por ejemplo Anemia australis, el complejo Anemia tomentosa, Anogramma lorentzii, Blechnum australe subsp. auriculatum y Cassebeera triphylla, reforzando la existencia del denominado Arco Peripampásico Serrano. Este patrón está apoyado además por la distribución de opiliones Ceratomontia centralis y $C$. argentina, halladas en las Sierras de Buenos Aires y también en Uruguay y el sur de Brasil, y por la de algunos escorpiones del género Bothriurus Peters, en especial de los grupos flavidus y prospicuus (Acosta 2002). La probable extensión del trazo hasta el noroeste argentino, tal cual lo establecido para los helechos, teniendo como nodo a las Sierras de Córdoba, también lo sugiere el rango de distribución de los escorpiones del grupo Bothriurus prospicuus (Acosta 2002) y la de los opiliones de los géneros Neopucroliella Roewer y Pachyloides Holmberg, estrechamente afines entre sí (Acosta 1993).

Las distribuciones de las floras del Cono Sur se vieron afectadas principalmente por fenómenos climáticos y geomorfológicos que comenzaron durante la segunda parte del Terciario: el levantamiento de los Andes, transgresiones oceánicas de corrientes frías, ciclos de aridez y disminución de la temperatura (Crisci et al. 2001). En los helechos en particular, la existencia de restos fósiles en el continente de paleoendemismos isleños actuales, sumado a las distribuciones disyuntas de géneros entre las áreas de endemismo y los centros de diversidad (por ejemplo Blechnum, Hymenophyllum, Ruhmora) indicarían que la flora de helechos de Argentina y Chile fue en el pasado más extensa y continua, siendo fragmentada $\mathrm{y}$ retrocediendo sus límites a causa de los cambios producidos por los fenómenos anteriormente mencionados (Ponce et al. 2002). Por otra parte, las glaciaciones del Pleistoceno ocasionaron impactantes fluctuaciones de aridez y humedad que causaron la fragmentación y diferenciación de las poblaciones (Villagrán \& Hinojosa 1997). En este período se extinguieron las floras subtropicales y se empobreció la flora de los bosques templados, avanzando la vegetación de zonas áridas y semiáridas en el continente, por ejemplo al norte de la Patagonia, el norte del desierto de Atacama, el Chaco y el Monte en el centro del cono sur de América del Sur, y el Cerrado y la Caatinga en el centro y noreste de Brasil (Prado \& Gibbs 1993). Estos fenómenos paleoclimáticos pueden haber provocado una separación de las poblaciones hacia las regiones montañosas y serranas, las que de acuerdo con Moran $(1995,2008)$ son de vital importancia para el establecimiento de helechos y licofitas porque poseen heterogeneidad de hábitats y microclimas. Algunos taxones se encuentran actualmente restringidos a las denominadas zonas intermedias (Ponce et al. 2002), en las cuales están incluidas las Sierras de Córdoba, en donde se ha mantenido una riqueza apreciable de helechos y licofitas, permaneciendo en estrecho intercambio florístico con las Sierras Subandinas y los Andes del Norte.

El patrón distribucional de los helechos de las sierras de la provincia de Córdoba muestra que la transición entre los sistemas serranos promovería la movilidad del componente biótico, migrando y colonizando los ambientes hasta que las condiciones climáticas y geográficas se lo permitan, ya que, por un lado, los helechos poseen una gran capacidad de dispersión debido a sus esporas livianas y ligeras, que se producen en gran cantidad, y por otro no existirían barreras apreciables entre los mencionados sistemas serranos, sino que los mismos conforman casi un continuo. Además, las Sierras Subandinas poseen una estrecha relación con los Andes del Norte, con los que no tiene ninguna barrera de separación específica. Para los helechos, y particularmente para licofitas, los sistemas serranos de Córdoba constituyen el extremo austral de un componente biótico neotropical ancestral, lo que se observa en el trazo generalizado obtenido por Arana et al. (2011). Por otra parte, debido a la barrera geográfica que significa la "Diagonal Árida de América del Sur”, la zona austral de Argentina y Chile está netamente separado de los centros de diversidad y áreas de endemismo del norte y centro, incluida la zona serrana de Córdoba, solamente elementos muy xeromorfos podrían atravesarla; mientras que las biotas del este y noroeste están separadas por las provincias biogeográficas del Chaco y la Pampa, principalmente por planicies cuya homogeneidad microambiental y períodos de aridez es un impedimento para el exitoso establecimiento de los helechos.

Dado que la conservación requiere preservar en un número mínimo de áreas la mayor diversidad posible, es necesario decidir cuáles áreas habrán de tener prioridad. Proteger un espacio geográfico físico, rico y complejo en especies, en el que habiten tanto taxones endémicos como de distribución más amplia, garantiza preservar las condiciones en las que los taxones se desenvuelven. En este sentido, el enfoque panbiogeográfico combinado con el PAE, presenta una síntesis entre sistemática, geología y ecología (Morrone \& Crisci 1995, Escalante 2003, Morrone 2009), resultando efectivo para detectar patrones de distribución y haciendo evidentes las regiones, que en términos de conservación, incluyen un conjunto representativo de la biodiversidad. Dentro de los cladogramas de áreas, los patrones de áreas anidadas en las ramas terminales son importantes para la determinación de áreas prioritarias para conservación (Posadas \& Miranda-Esquivel 1999), debido a que en ellas se concentra el mayor número de especies, las que a su vez se encuentran presentes en el resto de las áreas que conforman el clado.

Este trabajo constituye un aporte para la construcción del Atlas Biogeográfico del centro de Argentina, donde particularmente se demuestra que las sierras de Córdoba constituyen un nodo panbiogeográfico, lo que implica que 
es un área biogeográficamente compleja y con una riqueza apreciable en cuanto a especies de helechos y a orígenes históricos, constituyendo un centro de convergencia biótica, por lo tanto conforman un área prioritaria para conservar. Como queda sustentado por el patrón distribucional de las licofitas (Arana et al. 2011), y en este trabajo de los helechos, los sistemas serranos de Córdoba constituyen el extremo austral de una expresión de la biota neotropical, en el límite con la Zona de Transición Sudamericana. Además, con la construcción de los trazos se ha demostrado que varios taxones tienen en las sierras de Córdoba su límite austral de distribución total, entre los helechos encontramos a Adiantum camptorachis, Asplenium achalense, A. lilloanum, Hypolepis poeppigii y Phlebodium areolatum, entre las licofitas a Diphasiastrum thyoides y Lycopodium clavatum subsp. clavatum (Arana et al. 2011); mientras que sólo una especie de helecho, Blechnum hastatum, alcanzaría su límite septentrional en el área estudiada.

\section{AGRADECIMIENTOS}

A Ricardo Martori y Liliana Aun (UNRC) por todo el apoyo y consejos brindados para llevar a cabo este trabajo.

\section{BIBLIOGRAFÍA}

AcostA, L.E. 1993. Escorpiones y opiliones de la provincia de Córdoba (Argentina): diversidad y zoogeografía. Bulletin de la Société Neuchâteloise des Siences Naturelles 116(1): 11-17.

Acosta, L. E. 2002. Patrones zoogeográficos de los opiliones argentinos (Arachnida: Opiliones). Revista Ibérica de Aracnología 6: 69-84.

Aguilar-Aguilar, R. \& R. Contreras. 2001. La distribución de los mamíferos marinos de México: un enfoque panbiogeográfico. En: J. Llorente \& J.J. Morrone (eds.), Introducción a la biogeografía en Latinoamérica: Teorías, conceptos, métodos y aplicaciones. pp. 197-211. Facultad de Ciencias, UNAM, México, D.F.

Álvarez-Mondragón, E. \& J.J. Morrone. 2004. Propuesta de áreas para la conservación de aves de México, empleando herramientas panbiogeográficas e índices de complementariedad. Interciencia 92: 112-120.

Arana, M.D. 2012. Diversidad, distribución y vinculaciones de la flora de Helechos y Licofitas del las Sierras de Córdoba, Argentina. Tesis Doctoral, UNRC, Río Cuarto. 317 pp.

Arana, M., M. Ponce \& N. Vischi. 2004. Sinopsis de los helechos y grupos relacionados (Pteridophyta) de la provincia de Córdoba, Argentina. Boletín de la Sociedad Argentina de Botánica 39(1-2): 89-114.

Arana, M. \& C. Bianco. 2009. Pteridófitas del centro de Argentina. Editorial UNRC: Río Cuarto. 64 pp.

Arana, M. \& A. Oggero. 2009. Zanthoxylum armatum, su presencia en Argentina. Darwiniana 47(2): 335-338.

Arana, M.D., J.J. Morrone, M. Ponce \& A.J. Oggero. 2011.
Licofitas (Equisetopsida: Lycopodiidae) de las sierras centrales de Argentina: Un enfoque panbiogeográfico. Gayana Botánica 68(1): 14-21.

Arana, M. \& C. Bianco. 2011. Helechos y licofitas del centro de Argentina. Editorial UNRC: Río Cuarto. 84 pp.

ArCand, N.N. \& T.A. Ranker. 2008. Conservation biology. In: T.A. Ranker \& C. H. Haufler (eds.), The biology and evolution of ferns and lycophytes, pp: 257-283. Cambridge Univ. Press, New York.

BARRINGTON, D.S. 1993. Ecological and historical factors in fern biogeography. Journal of Biogeography 20: 275- 280.

Bertonatti, C. \& J. Corcuera. 2000. Situación Ambiental Argentina 2000. Fundación Vida Silvestre, Buenos Aires. 440 pp.

Bianco C.A., T.A. Kraus, D.L. Anderson \& J.J. Cantero. 1987. Formaciones vegetales del suroeste de la provincia de Córdoba (República Argentina). Revista UNRC 7(1): 566.

Cabrera, A.L. \& A. Willink. 1973. Biogeografía de América Latina. Monografía 13, Serie de Biología, OEA. Washington, D.C. 117 pp.

Capurro, R. 1969. División Pteridophyta. En. A.L. Cabrera (ed.), Flora de la Provincia de Buenos Aires. Colección del Instituto Nacional de Tecnología Agropecuaria 4: 123-146.

Chase, M.W. \& J.L. Reveal. 2009. A phylogenetic classification of the land plants to accompany APG III. Botanical Journal of the Linnean Society 161: 122-127.

Christenhusz, M.J.M., X. Zhang \& H. Schneider. 2011. A linear sequence of extant families and genera of lycophytes and ferns. Phytotaxa 19: 7-54.

CEI, J.M. 1980. Amphibians of Argentina. Monitore zoologico Italiano (Nova Serie) Monographies 2: 1-609.

CeI, J.M. 1986. Reptiles del centro, centro-oeste y sur de la Argentina. Herpetofauna de las zonas áridas y semiáridas. Museo Regionali di Scienze Naturali, Torino, Italia. 527 pp.

Contreras-Medina, R. 2006. Los métodos de análisis biogeográficos y su aplicación a la distribución de las gimnospermas de México. Interciencia 31: 176-182.

Contreras-Medina, R., J.J. Morrone \& I. Luna. 2001. Biogeographic methods identify gymnosperm biodiversity hotspots. Naturwissenschaften 88: 427-430.

Craw, R.C. 1988. Panbiogeography: Method and synthesis in biogeography. In: A.A. Myers \& P.S. Giller (eds.), Analytical biogeography: An integrated approach to the study of animal and plant distributions, pp 405-435. Chapman \& Hall: Londres \& Nueva York.

Craw, R.C., J.R. Grehan \& M.J. Heads. 1999. Panbiogeography: Tracking the history of life. Oxford Biogeography Series no. 11. Oxford University Press, Oxford. 240 pp.

Crisci, J.V. 2006. Espejos de nuestra época: Biodiversidad, sistemática y educación. Gayana Botanica 63(1): 106-114.

Crisci, J.V., L. Katinas \& P. Posadas. 2000. Introducción a la teoría y práctica de la biogeografía histórica. Sociedad Argentina de Botánica. Buenos Aires. 169 pp.

Crisci, J.V., S.E. Freire, G. Sancho \& L. Katinas. 2001. Historical biogeography of the Asteraceae from Tandilia and Ventania Mountain ranges. Caldasia 23(1): 21-41.

De la Sota, E.R. 1967. Composición, origen y vinculaciones de la flora pteridológica de las sierras de Buenos Aires 
(Argentina). Boletín de la Sociedad Argentina de Botánica 11(2-3): 105-128.

Dela Sota, E.R. 1972. Notas sobre las especies austrosudamericanas del género Blechnum L., III, El género Blechnum en la provincia de Buenos Aires. Boletín de la Sociedad Argentina de Botánica 14: 177-197.

De la Sota, E.R. 1973. La distribución geográfica de las pteridófitas en el cono sur de América meridional. Boletín de la Sociedad Argentina de Botánica 15(1): 23 - 34.

De la Sota, ER. 1977. Pteridophyta, en A. L. Cabrera (Ed.). Flora de la provincia de Jujuy, Tomo XIII parte II. pp. 1-275. INTA. Buenos Aires.

De la Sota, E.R. 1985. Las pteridofitas de la provincia de La Pampa, Argentina. Revista de la Facultad de Agronomía U. N. La Pampa 1(1-2): 23-34.

De la Sota, E.R., M.M. Ponce, M.A. Morbelli \& L.A. Cassá DE Pazos. 1998. Pteridophyta. En: M.N. Correa (ed.), Flora Patagónica, Tomo VIII, parte I. pp. 282-370. INTA, Buenos Aires.

De la Sota, E.R., M.L. Luna, G.E. Giudice \& J.P. Ramos Giacosa. 2009. Sinopsis de las Pteridofitas de la provincia de San Luis (Argentina). Boletín de la Sociedad Argentina de Botánica 44: 367-385.

De Pinna, M.C.C. 1991. Concepts and tests of homology in the cladistic paradigm. Cladistics 7: 367-394.

Del Moral Sachetti, J.F. \& F.I. Lameda Camacaro. 2011. Registros de ocurrencia del oso andino (Tremarctos ornatus Cuvier, 1825) en sus límites de distribución nororiental y austral. Revista del Museo Argentino de Ciencias Naturales nueva serie 13(1): 7-19.

Dirzo, R. \& P.H. Raven. 2003. Global state of biodiversity and loss. Annual Review of Environment and Resources 28: 137-167.

Escalante, T. 2003. Avances en el atlas biogeográfico de los mamíferos terrestres de México. En: J.J. Morrone \& J. Llorente (eds.), Una perspectiva latinoamericana de la biogeografía. pp. 297-302. Las Prensas de Ciencias, UNAM. México, D.F.

Frenguelli, J. 1950. Rasgos generales de la morfología y la geología de la provincia de Buenos Aires. Serie 2, n 33, LEMIT. La Plata. 72 pp.

Goloboff, P.A. 1998. Principios básicos de cladística. Sociedad Argentina de Botánica, Buenos Aires. 81 pp.

Grehan, J.R. 1993. Conservation biogeography and the biodiversity crisis: A global problem in space/time. Biodiversity Letters 1: 134-140.

Halffter, G. 2000. Medir la biodiversidad. En: F. Martín-Piera, J.J. Morrone \& A. Melic (eds.), Hacia un proyecto CYTED para el inventario y Estimación de la Diversidad Entomológica en Iberoamérica: PrIBES 2000. m3mmonografías Tercer Milenio, vol. 1 pp11-18. Sociedad Entomológica Aragonesa (SEA), Zaragoza.

Heads, M. 2004. What is a node? Journal of Biogeography 31: 1883-1891.

Hicken, C. 1908. Polypodiacearum Argentinarum Catalogus. Revista del Museo de La Plata, Sección Botánica 15: 226282.

Hicken, C.M. 1919. La migración de los helechos en la flora de Tucumán. Primera Reunión Nacional de la Sociedad Argentina de Ciencias Naturales, San Miguel de Tucumán. pp. 187-209.

Hieronymus, G. 1896. Beitrage zur Kenntnis der Pteridophyten Flora der Argentina und einiger agrenzender Teile von Uruguay, Paraguay und Bolivien. Botanische Jahrbücher fur Systematik 22: 359-420.

Kessler, M. 2010. Biogeography of ferns. In: K. Mehltreter, L.R. Walker \& J.M. Sharpe (eds.), Fern ecology. pp: 22-60. Cambridge University Press. Cambridge.

Lindenmayer, D.B., C.R. Margules \& D.B. Botkin. 2000. Indicators of Biodiversity for Ecologically Sustainable Forest Management. Conservation Biology 14(4): 941950.

Luti, R., M.A.B. Solis, F.M. Galera, N.M. Ferreyra, M. Brezal, M. Nores, M.A. Herrera \& J.C. Barrera. 1979. Vegetación. En: J.B. Vazquez, R.A. Miatello \& M.E. Roqué (dirs.), Geografía física de la provincia de Córdoba. Pp: 297- 368. Ediciones Boldt, Buenos Aires.

Mace, G.M. 2004. The role of taxonomy in species conservation. Philosophical Transactions of the Royal Society of London B 359: 711-719.

Martínez-Aquino, A., R. Aguilar-Aguilar, H.O. Santa Anna del Conde-Juárez \& R. Contreras-Medina. 2007. Empleo de herramientas panbiogeográficas para detectar áreas para conservar: Un ejemplo con taxones dulceacuícolas. En: I. Luna, J.J. Morrone \& D. Espinosa (eds.), Biodiversidad de la Faja Volcánica Transmexicana. Pp: 449-460. Facultad de Ciencias, Universidad Nacional Autónoma de México. México, D.F.

McNeely, J.A. 2002. The role of taxonomy in conserving biodiversity. Journal for Nature Conservation 10: 145-153.

Moran, R.C. 1995. The importance of mountains to Pteridophytes, with emphasis on Neotropical montane forests. In: S.P. Churchill (ed.), Biodiversity and conservation of Neotropical montane forests. Pp 359- 363. New York Botanical Garden. New York.

Moran, R.C. 2008. Diversity, biogeography and floristics. In: T.A. Ranker \& C.H. Haufler (eds.), The biology and evolution of ferns and lycophytes. Pp: 367-394. Cambridge University Press, New York.

Moré, M., I. Kitching \& A.A. Cocucci. 2005. Sphingidae: Esfíngidos de Argentina. Hawkmoths of Argentina. Editorial L.O.L.A., Buenos Aires. 166 pp.

Morrone, J.J. 2000a. What is the Chacoan Region? Neotropica 46: 51-68.

Morrone, J.J. 2000b. La importancia de los atlas biogeográficos para la conservación de la biodiversidad. En: F. MartínPiera, J.J. Morrone, A. Melic (eds.), Hacia un proyecto CYTED para el inventario y estimación de la diversidad entomológica en Iberoamérica: PrIBES. Vol. 1. pp. 69-78. SEA-CYTED-Instituto Humboldt, Monografías Tercer Milenio. Zaragoza.

Morrone, J.J. 2001a. Sistemática, biogeografía, evolución: Los patrones de la biodiversidad en tiempo-espacio. Las Prensas de Ciencias. UNAM. México, D.F. 124 pp.

Morrone, J.J. 2001b. Biogeografía de América Latina y el Caribe. Manuales \& tesis SEA. Zaragoza. 148 pp.

Morrone, J.J. 2004. La zona de transición sudamericana: Caracterización y relevancia evolutiva. Acta Entomologica Chilena 28: 41-50.

Morrone, J.J. 2006. Biogeographic areas and transition zones 
Biogeografía y conservación de helechos de la Argentina central: ArANA, M. ET.AL.

of Latin American and the Caribbean islands based on panbiogeographic and cladistic analyses of the entomofauna. Annual Review of Entomology 51: 467-494.

Morrone, J.J. 2007. Hacia una biogeografía evolutiva. Revista Chilena de Historia Natural 80: 509-520.

Morrone, J.J. 2009. Evolutionary biogeography: An integrative approach with case studies. Columbia University Press, Nueva York, $301 \mathrm{pp}$.

Morrone, J.J. \& J.V. Crisci. 1995a. Aplicación de métodos filogenéticos y panbiogeográficos en la conservación de la diversidad biológica. Evolución Biológica (Bogotá) 6: 53-66.

Morrone, J.J. \& J.V. Crisci. 1995b. Historical biogeography: Introduction to methods. Annual Review of Ecology and Systematics 26: 373-401.

Morrone, J.J. \& D. Espinosa. 1998. La relevancia de los Atlas Biogeográficos para la conservación de la biodiversidad mexicana. Ciencia 49: 12-16.

NovaceK, M.J. 2008. Engaging the public in biodiversity issues. Proceedings of the National Academy of Sciences 105(Suppl. 1): 11571-11578.

OchoA, J.A. 2005. Patrones de distribución de escorpiones de la región andina en el sur peruano. Revista Peruana de Biología 12(1): 49-68.

Oggero, A.J. \& M.D. Arana. 2012. Inventario de la Biodiversidad de Plantas Vasculares del sur de la zona serrana de Córdoba, Argentina. Hoehnea 39(2): 169- 197.

O’Leary, N., P. Peralta \& M.E. Múlgura. 2011. Sinopsis del género Junellia (Verbenaceae). Darwiniana 49(1): 47-75.

PAge, C.N. 1985. Pteridophyte Biology: the biology of amphibians of the plant world. Proceedings of the Royal Society of Edinburgh. Section B. Biological Sciences. 86 B: 439-442.

Pastore, A. 1936. Las Isoetáceas argentinas. Revista del Museo de La Plata, Nueva Serie 1: 1-30.

Pirani, J.R. \& J. Prado. 2012. Embryopsida, a new name for the class of land plants. Taxon 61:1096-1098.

Ponce, M.M. 1996. Pteridophyta. En: F.O. Zuloaga \& O. Morrone (eds.), Catálogo de las plantas vasculares de la República Argentina I: Pteridophyta, Gymnospermae y Angiospermae (Monocotyledoneae). Monographs in Systematic Botany of the Missouri Botanical Garden 60: 1-79.

Ponce, M., K. Mehltreter \& E.R. De la Sota. 2002. Análisis biogeográfico de la diversidad pteridofítica en Argentina y Chile continental. Revista Chilena de Historia Natural 75: 703-717.

Posadas, P. \& D. Miranda-Esquivel. 1999. El PAE (Parsimony Analysis of Endemicity) como una herramienta en la evaluación de la biodiversidad. Revista Chilena de Historia Natural 72: 539-546.

Prado, D.E. \& P.E. GibBs. 1993. Patterns of species distributions in the dry seasonal forests of South America. Annals of the Missouri Botanical Garden 80: 902-927.

Prado, J.L., J. Chiesa, G. Tognelli, E. Cerdeño \& E. Strasser. 1998. Los mamíferos de la formación Río Quinto (Plioceno), provincia de San Luis (Argentina). Aspectos bioestratigraficos, zoogeograficos y paleoambientales. Estudios Geológicos 54: 153-160.

RAmos, V.A. 1999. Las provincias geológicas del territorio argentino. Geología Argentina-Anales 29 (3): 41-96.

RAVEN, P.H. \& D.I. AxeLroD. 1972. Plate tectonics and Australasian paleobiogeography. Science 176: 1379-1386.

Rodríguez, R. 1995. Pteridophyta. En: C. Marticorena \& R. Rodríguez Ríos (eds.), Flora de Chile, Volumen I: 119-337. Ediciones de la Universidad de Concepción, Concepción.

Sanmartín, I. \& F. Ronquist. 2004. Southern Hemisphere Biogeography inferred by event-based models: Plant versus Animal patterns. Systematic Biology 53(2): 216243.

Schiavone, M.M. \& G.M. SuÁrez. 2007. Las Thuidiaceae en el Noroeste de Argentina Boletín de la Sociedad Argentina de Botánica 42(3-4): 211 - 230.

Schneider, H., K.M. Pryer, R. Cranfill, A.R. Smith \& P.G. WoLf. 2002. Evolution of vascular plant body plans: a phylogenetic perspective. In: Q.C.B. Conk, R.M. Bateman \& J.A. Hawkins (eds.), Developmental Genetics and Plant Evolution, pp. 330-364. Taylor \& Francis, Londres.

Schneider, H., E. Schuettpelz, K.M. Pryer, R. Cranfill, S. Magallón \& R. Lupia. 2004. Ferns diversified in the shadow of angiosperms. Nature 428: 553- 557.

SINGH, J.S. 2002. The biodiversity crisis: A multifaceted review. Current Science 82(6): 638-647.

Smith, A.R., K.M. Pryer, E. Schuettpelz, P. Korall, H. Schneider \& P.G. Wolf. 2006. A classification for extant ferns. Taxon 55(3): 705-731.

Sмiтh, L.B. 1974. Bromeliaceae subfamily Pitcairnioideae. En: L. B. Smith \& R. J. Downs, Pitcairnioideae. Flora Neotropica Monograph 14(1): 1-662.

Squeo, F.A., L.A. Cavieres, G. Arancio, J.E. Novoa, O. Matthei, C. Marticorena, R. Rodríguez, M.T.K. Arroyo \& M. MuÑoz. 1998. Biodiversidad de la flora vascular en la región de Antofagasta, Chile. Revista Chilena de Historia Natural 71: 571- 591.

Swenson, U., R.S. Hill \& S. Mc Loughlin. 2001. Biogeography of Nothofagus supports the sequence of Gondwana break-up. Taxon 50: 1025- 1041.

Swofford, D.L. 1999. PAUP*: Phylogenetic analysis using parsimony. Version 4.0 beta 2. Sinauer Associates, Sunderland. Massachusetts.

ThIERS, B.M. (ED.) 2013. Index Herbariorum. New York Botanical Garden, New York. (http://sciweb.nybg.org/science2/ IndexHerbariorum.asp).

Tryon, R.M. \& A.F. Tryon. 1982. Ferns and allied plants, with special reference to Tropical America. Springer-Verlag, New York, Heidelberg y Berlín.

Van Bocxlaer, I., K. Roelants, S.D. Biju, J. Nagaraju \& F. Bossuyt. 2006. Late Cretaceous Vicariance in Gondwanan Amphibians. PLoS ONE 1(1): e74. doi:10.1371/journal. pone. 0000074 .

Villagrán, C. \& L.F. Hinojosa. 1997. Historia de los bosques de Sudamérica II: Análisis fitogeográfico. Revista Chilena de Historia Natural 70: 241-267.

Verzino, G., J. Joseau, M. Dorado, E. Gellert, S. Rodriguez Reartes \& R. Nóbile. 2005. Impacto de los incendios sobre la diversidad vegetal, Sierras de Córdoba, Argentina. Ecología Aplicada 4(1-2): 25-34.

Vischi, N.B., A.J. OgGero \& M.D. Arana. 2001. Reserva florística "Las Guindas". Estudio previo a su establecimiento. Revista UNRC 19(1-2): 37-55.

Weberling, F. 1999. Movimiento de los continentes y separación e intercambio de sus floras. Sociedad Argentina de Botánica, 
Buenos Aires. 47 pp.

Wright, A. 2004. "Leopardus jacobitus", Animal Diversity Web. 2012. Accessed April 18. www.animaldiversity. ummz.umich.edu/site/accounts/information/Leopardus jacobitus.html.

ZaK, M., M. CABido, D. CÁceres \& S. Díaz. 2008. What drives accelerated land cover change in central Argentina? Environmental Management 42: 181-189.

Zunino, M. \& A. Zullini. 2003. Biogeografía: La dimensión espacial de la evolución. Fondo de Cultura Económica, México, D.F. 359 pp.

ApÉNDICE 1: Clasificación y secuencia lineal de los helechos presentes en la provincia de Córdoba (Argentina).

APPENDIX 1: Classification and linear sequence of ferns from Cordoba province (Argentina).

División Charophyta Migula

Clase: Embryopsida Engler ex Pirani \& J. Prado

Subclase Ophioglossidae Klinge

Orden Ophioglossales Link

Familia Ophioglossaceae Martynov

Botrychium australe R. Br. subsp. australe (5)

Ophioglossum crotalophoroides Walter ssp. crotalophoroides (5)

O. crotalophoroides Walter var. nanum J.S. Licht. ex Osten (5)

Subclase Equisetidae Warm.

Orden Equisetales DC. ex Bercht. \& J. Presl

Familia Equisetaceae Michx. ex DC.

Equisetum bogotense Kunth (5)

E. giganteum L. (6)

Subclase Polypodiidae Cronquist, Takht. \& Zimmerm.

Orden Hymenophyllales A.B. Frank

Familia Hymenophyllaceae Mart.

Hymenophyllum tunbrigense (L.) Sm. var. cordobense Hieron. (*)

Orden Schizaeales Schimp.

Familia Anemiaceae Link

Anemia australis (Mickel) M. Kessler \& A.R. Sm. (*, 6)

A. tomentosa (Savigny) Sw. var. anthriscifolia (Schrad.) Mickel (5)

A. tomentosa (Savigny) Sw. var. tomentosa (6)

Orden Salviniales Bartl.

Familia Marsileaceae Mirb.

Marsilea ancylopoda A. Braun (5)

Pilularia americana A. Braun (5)

Familia Salviniaceae Martynov

Azolla filiculoides Lam. (6)

Orden Polypodiales Link

Familia Dennstaedtiaceae Lotsy

Dennstaedtia globulifera (Poir.) Hieron. (*, 6)

Hypolepis poeppigii (Kunze) R. A. Rodr. $(*, 1)$

Pteridium arachnoideum (Kaulf.) Maxon (*, 6)

Familia Pteridaceae E.D.M. Kirchn.

Adiantopsis chlorophylla (Sw.) Fée (6)

A. tweediana (Hook.) Link-Pérez \& Hickey (5)

Adiantum camptorachis Sundue, J. Prado \& A. R. Sm. (*)

A. lorentzii Hieron. (5)

A. orbignyanum Mett. ex Kuhn $(*, 2,6)$

A. raddianum C. Presl. (5)

A. thalictroides Willd. ex Schltdl. f. bottini Giúdice \& Nieto (*, 5)

A. thalictroides Willd. ex Schltdl. var. thalictroides (5)

Anogramma chaerophylla (Desv.) Link

A. lorentzii (Hieron.) Diels

Argyrochosma nivea (Poir.) Winham var. flava (Hook.) Ponce (*)

A. nivea (Poir.) Winham var. nivea $(*)$ 
A. nivea (Poir.) Winham var. tenera (Gill. ex Hook.) Ponce (*, 5)

Cassebeera triphylla (Lam.) Christ. (5)

Cheilanthes bonariensis (Willd.) Proctor $(*, 5)$

C. buchtienii (Rosenst.) R. M. Tryon (*, 5)

C. marginata H.B.K. $(*, 5)$

C. micropteris Sw. $(*)$

C. myriophylla Desv. (*)

C. obducta Mett. ex Kuhn (5)

C. pilosa Goldm. (*, 2)

C. pruinata Kaulf. (*)

C. squamosa Gillies ex Hook. \& Grev. (*, 3, 5)

Doryopteris concolor (Langsd. et Fisch.) Kuhn (6)

D. lorentzii (Hieron.) Diels (5)

Notholaena sulphurea (Cav.) J.Sm. (*, 5)

Pellaea ternifolia (Cav.) Link. (*)

Pityrogramma calomelanos (L.) Link var. austroamericana (Domin.) Farw. (*, 5)

P. calomelanos (L.) Link var. calomelanos (5)

P. tartarea (Cav.) Maxon var. aurata (Moore) R. M. Tryon (*, 5)

Trismeria trifoliata (L.) Diels $(*, 6)$

Familia Cystopteridaceae (Payer) Schmakov

Cystopteris diaphana (Bory) Blasdell (5)

Familia Aspleniaceae Newman

Asplenium achalense Hieron. (*, 1)

A. depauperatum Fée (5)

A. gilliesii Hook. (*, 3)

A. lilloanum de la Sota $(*, 1)$

A. lorentzii Hieron. (*, 2, 5)

A. monanthes L. (6)

A. resiliens Kunze (*, 5)

Familia Thelypteridaceae Ching ex Pic. Serm.

Thelypteris achalensis (Hieron.) Abbiatti (*)

T. argentina (Hieron.) Abbiatti (*)

Familia Woodsiaceae Herter

Woodsia montevidensis (Spreng.) Hieron.

Familia Blechnaceae Newman

Blechnum australe L. ssp. auriculatum (Cav.) de la Sota (*)

B. cordatum (Desv.) Hieron.

B. hastatum Kaulf.

B. laevigatum Cav.

B. mochaenum G. Kunkel var. achalense (Hieron.) de la Sota $(*, 5)$

B. penna-marina (Poir.) Kuhn (5)

B. squamipes (Hieron.) M. Kessler \& A.R. Sm. (*, 1)

B. x pampasicum Sota \& Duran (5)

Familia Athyriaceae Alston

Athyrium dombeyi Desv. (*, 4)

Familia Dryopteridaceae Herter nom. cons.

Elaphoglossum gayanum (Fée) T. Moore (6)

E. lorentzii (Hieron.) H. Christ. $(*, 5)$

E. piloselloides (C. Presl) T. Moore $(*, 5)$

Polystichum montevidense (Spreng.) Rosenst. var. montevidense (*, 4, 6)

P. montevidense (Spreng.) Rosenst. var. squamulosa (Hieron.) Hieron. $(*, 5)$

Familia Polypodiaceae J. Presl

Melpomene peruviana (Desv.) A.R. Sm. \& R.C. Moran (*)

Microgramma vacciniifolia (Langsd. \& Fisch.) Copel. (6)

Phlebodium areolatum (Willd.) J. Sm. (*,6)

Pleopeltis minima (Bory) J. Prado \& R. Y. Hirai

P. pinnatifida Gillies ex Hook. \& Grev. $(*, 6)$

Serpocaulon gilliesii (C.Chr.) A.R. Sm. $(*, 5)$ 
APÉNDICE 2: Material representativo examinado proveniente del área de estudio

APPENDIX 2: Representative material examined from the study area.

\section{OPHIOGLOSSACEAE}

Botrychium australe $\mathrm{R}$. Br. subsp. australe

Córdoba. Dpto. Río Cuarto. Las Guindas, pastizal de altura, Arana s/n (RCV 3359), entre Achiras y La Punilla, Bianco-Cantero 2146 (RIOC).

Ophioglossum crotalophoroides Walter subsp. crotalophoroides Córdoba. Dpto. Calamuchita. Quebrada del Rayo, pastizal de altura, Bianco et al. (2517 RIOC).

Ophioglossum crotalophoroides Walter var. nanum J.S. Licht. ex Osten

Córdoba. Dpto Punilla. Sierra Grande, 24-XI-1932, más arriba de Copina, C.C. Hosseus 23 (Paratipo CORD).

\section{EQUISETACEAE}

Equisetum bogotense Kunth

Córdoba. Dpto. Calamuchita. Santa Rosa de Calamuchita, Zavala $\mathrm{s} / \mathrm{n}(\mathrm{RCV})$.

Equisetum giganteum L.

Córdoba. Dpto. Río Cuarto. Alto Lindo, Las Albahacas, Vischi s/n (RCV 936).

HYMENOPHYLLACEAE

Hymenophyllum tunbrigense (L.) Sm. var. cordobense Hieron. Córdoba. Dpto. Río Cuarto. Mesada de Crespo, pastizal de altura, Bianco 2134 (RIOC).

\section{ANEMIACEAE}

Anemia australis (Mickel) M. Kessler \& A.R. Sm.

Córdoba. Dpto. Calamuchita. Segunda Usina, Bocco et al. s/n (RCV 906).

\section{Anemia tomentosa (Savigny) Sw. var. tomentosa}

Córdoba. Dpto. Calamuchita. Segunda Usina, Bocco s/n (RCV 770).

Anemia tomentosa (Savigny) Sw var. anthriscifolia (Schrad.) Mickel

Córdoba. Dpto. Santa María. Río Anizacate, cerca de 10 km de Alta Gracia, 17-I-1940, A. T. Hunziker 3572 (CORD).

\section{MARSILEACEAE}

Marsilea ancylopoda A. Braun

Córdoba. Dpto. Calamuchita. Proximidades Villa Rumipal, X-2012, C. A. Bianco s.n. (RIOC).

Pilularia americana A. Braun

Córdoba: Dpto. Punilla. Sierra de Achala, Estancia San Miguel (Hieronymus $\mathrm{s} / \mathrm{n}$ ).

\section{SALVINIACEAE}

Azolla filiculoides Lam.

Córdoba. Dpto. Río Cuarto. Río Cuarto, Bianco 2293 (RIOC).

\section{DENNSTAEDTIACEAE}

Dennstaedtia globulifera (Poir.) Hieron.

Córdoba. Dpto. Punilla: Casa Bamba, "in valleculo húmedo copioso”, 14/VI/1907, Kurtz 13097 (CORD).

Hypolepis poeppigii (L.) R. Rodr.

Córdoba. Dpto. Calamuchita: Sa. de Comechingones, Alpa Corral, romerillal, Arana s/n. (RCV 3604, SI).

Pteridium arachnoideum (Kaulf.) Maxon

Córdoba. Dpto. Punilla. Pampa de Achala, pastizal Arana s/n (RCV 3711).

\section{PTERIDACEAE}

Adiantopsis chlorophylla (Sw.) Fée

Córdoba. Dpto. Calamuchita. Las Guindas, Sa. de Comechingones, pastizal, Arana $546(\mathrm{RCV})$.

Adiantopsis tweediana (Hook.) Link-Pérez \& Hickey

Córdoba: Dpto. Punilla. Capilla del Monte, en lugares húmedos de El Zapato, 3-X-1918, Castellanos 730 (SI).

Adiantum camptorachis Sundue, J. Prado \& A. R. Sm.

Córdoba. Dpto. Calamuchita. Lutti, Bianco 2294 (RIOC). Dpto. Santa María. Pampa de Achala, Hosseus 152 (CORD).

Adiantum lorentzii Hieron.

Córdoba. Dpto. Punilla. Pampa Pampa de Achala, pastizal Arana s/n (RCV 3703).

Adiantum orbignyanum Mett. ex Kuhn

Córdoba. Dpto. Calamuchita. El Cano, Bianco \& Cantero s/n (RCV 2175).

Adiantum raddianum $\mathrm{C}$. Presl

Córdoba. Dpto Río Cuarto. Alpa Corral, Bocco s/n (RCV 1640).

Adiantum thalictroides Willd. ex Schltdl. var. thalictroides Córdoba. Dpto. Punilla. Pampa de Achala, pastizal Arana s/n (RCV 3721).

Adiantum thalictroides Willd. ex Schltdl. f. bottini Giudice \& Nieto

Córdoba. Dpto. Calamuchita. Las Guindas, pastizal, Arana s/n (RCV 3358).

Anogramma chaerophylla (Desv.) Link

Córdoba. Dpto. Punilla. Sierra de Achala, N de Cuesta de Copina, 12/13-XI-1878, Hieronymus s/n.

Anogramma lorentzii (Hieron.) Diels

Córdoba. Depto. Calamuchita. Sierra de Comechingones, falda este, cerca del pie del cerro Champaquí, 14-III-1951, A. T. Hunziker 9069 (CORD). 
Biogeografía y conservación de helechos de la Argentina central: ArANA, M. ET.AL.

Argyrochosma nivea (Poir.) Winham var. nivea

Córdoba. Dpto. Río Cuarto. Villa El Chacay, Bianco-Cantero 801 (RIOC).

Argyrochosma nivea (Poir.) Winham var. flava (Hook.) Ponce Córdoba. Dpto. Calamuchita. Segunda Usina, Bocco s/n (958 $\mathrm{RCV})$.

Argyrochosma nivea (Poir.) Winham var. tenera (Gillies ex Hook.) Ponce

Córdoba. Dpto. Río Cuarto. Entre Achiras y La Punilla, Bianco \& Cantero 2125 (RIOC).

Cassebeera triphylla (Lam.) Kaulf.

Córdoba: Dpto. Tulumba. Cerro Colorado, Arana s/n (RCV 2875).

Cheilanthes bonariensis (Willd.) Proctor

Córdoba. Dpto. Río Cuarto. Proximidades del límite interprovincial entre San Luis y Córdoba, a la altura de Papagayos, BiancoCantero 2120 (RIOC).

Cheilanthes buchtienii (Rosenst.) R.M. Tryon

Córdoba. Dpto. Punilla. Pampa de Achala, pastizal, Arana s/n (RCV 3722).

\section{Cheilanthes marginata Kunth}

Córdoba. Dpto. Punilla. Sierra de Achala, Puerto Alegre, 5-III1877, Hieronymus 872 (CORD).

\section{Cheilanthes micropteris Sw.}

Córdoba. Dpto. Calamuchita. Segunda Usina, Bocco et al. s/n (RCV 1105).

\section{Cheilanthes myriophylla Desv.}

Córdoba. Dpto Capital. Ciudad de Córdoba, Altos Crisol, 31XII-1896, Stuckert 1192 (CORD). Dpto Río Cuarto. Alpa Corral, bosque serrano, Ceballos s/n (3335 RCV).

Cheilanthes obducta Mett. ex Kuhn

Córdoba. Dpto. Calamuchita. Yacanto, I-1922, Vattuone 2 (SI); San Marcos, 18-IV-1940, E. Nicora 2791 (SI). Dpto. Cruz del Eje. Crespo, 28-II-1899, Stuckert 6582 (CORD).

\section{Cheilanthes pilosa Goldm.}

Córdoba: Dpto. Punilla. Capilla del Monte, mogote, 1-II-1917, Hosseus 127 (CORD). Dpto. Rio Cuarto. Cerro Inti Huasi Sa. de Comechingones, bosque, Ceballos s/n (3535 RCV).

\section{Cheilanthes pruinata Kaulf.}

Córdoba: Dpto. Punilla. Sierra de Achala, Cuesta del Tránsito, in fissuri rupium, 6/13-I-1895, F. Kurtz 8344 (CORD, SI).

\section{Cheilanthes squamosa Gillies ex Hook. \& Grev.}

Córdoba: Dpto. Colón. Unquillo, 1-IX-1918, Hosseus 130 (CORD). Dpto. Punilla. Los Gigantes, 1-V-1933, Hosseus 50a (CORD).

Doryopteris concolor (Langsd. \& Fisch.) Kuhn Córdoba: Dpto. Calamuchita. Las Guindas, Sa. de Comechingones, pastizal, Arana s/n (2893 RCV).
Doryopteris lorentzii (Hieron.) Diels

Córdoba. Dpto. Capital. Ciudad de Córdoba, río Primero abajo, puesto del paraíso, in declivibus silvatis passin, 10-II-1899, Kurtz 10348 (CORD).

Notholaena sulphurea (Cav.) J. Sm.

Córdoba. Dpto Punilla. Cumbre del Overo, Capilla del Monte, 30I-1917, Hosseus 174 (CORD).

Pellaea ternifolia (Cav.) Link

Córdoba: Dpto. Calamuchita. Las Guindas, Sa. de Comechingones, pastizal, Arana s/n (2107 RCV).

Pityrogramma calomelanos (L.) Link var. austroamericana (Domin) Farw.

Córdoba: Dpto. Punilla. Villa García, 16-XII-1909, Stuckert 20556 (CORD).

Pityrogramma calomelanos (L.) Link var. calomelanos Córdoba: Dpto. Tulumba. Cerro Colorado, Arana s/n (2862 RCV).

Pityrogramma tartarea (Cav.) Maxon var. aurata (Moore) R. M. Tyron

Córdoba. Dpto. Punilla. Carlos Paz, Sierra de Córdoba, Bridarolli 298, 809 (LP); Cuesta del Tránsito, Kurtz 8358 (LP).

Trismeria trifoliata (L.) Diels

Córdoba: Dpto. Calamuchita. La Falda, 29-I-1904, Stuckert 16948 (CORD). Dpto. Punilla. Paso de la Higuera, Sierra Grande (Achala), 24/25-III-1875, Hieronymus 222 (CORD).

\section{CYSTOPTERIDACEAE}

Cystopteris diaphana (Bory) Blasdell

Córdoba: Dpto. Calamuchita. Cerro Áspero, Salto del tigre, 21-III2009, Mealla s/n (RCV).

\section{ASPLENIACEAE}

Asplenium achalense Hieron.

Córdoba. Dpto. Punilla. Córdoba, Sierra de Achala, Tanti Cuchi (del Rosario), 12-II-1876, G. Hieronymus 846 (Sintipo, CORD, Isosintipo, SI).

Asplenium depauperatum Fée

Córdoba: Dpto. Río Seco. $12 \mathrm{~km}$ al norte de Villa de María, poco antes de San Miguel, abundante a la sombra de roca, 7-XI-1949, A. T. Hunziker 8007 (CORD).

Asplenium gilliesii Hook.

Córdoba: Dpto. Calamuchita. Las Guindas, Sa. de Comechingones, pastizal, Arana s/n (3521 RCV). Dpto. San Alberto. Sierra Grande, Pampa de Achala, cerca del Hotel La Posta, 13/14-XII-1945, A. T. Hunziker 6465 (CORD).

Asplenium lilloanum de la Sota

Córdoba: Dpto. Calamuchita. Santa Rosa de Calamuchita, Sa. de Comechingones, bosque, Zavala s/n (4071 RCV).

Asplenium lorentzii Hieron.

Córdoba: Dpto. Colón. Ascochinga, abundante en cañadón, en el monte, 8-VII-1938, E. G. Nicora 1970 (SI). 
Asplenium monanthes L.

Córdoba: Dpto. Calamuchita. Las Guindas, Sa. de Comechingones, pastizal, Ceballos s/n (3652 RCV).

Asplenium resiliens Kunze

Córdoba: Dpto. Calamuchita. Embalse, Bosque serrano, Arana s/n (2792 RCV).

\section{THELYPTERIDACEAE}

Thelypteris achalensis (Hieron.) Abbiatti

Córdoba: Dpto. Punilla. Quebrada al pié de Los Gigantes, sierra de Achala, 24-I-1880, Galander s/n (CORD)

Thelypteris argentina (Hieron.) Abbiatti

Córdoba: Dpto. Calamuchita. Lutti, Bianco-Cantero 2171 (RIOC).

\section{WOODSIACEAE}

Woodsia montevidensis (Spreng.) Hieron.

Córdoba: Dpto. Punilla. Pampa de Achala, pastizal, Arana s/n (3727 RCV).

\section{ATHYRIACEAE}

Athyrium dombeyi Desv.

Córdoba Dpto. Punilla. Pampa de Achala, $2200 \mathrm{~m}$, en hueco formado por peñascos, 13-XII-1945, J.H. Hunziker 1392 (SI).

\section{BLECHNACEAE}

Blechnum australe L. ssp. auriculatum (Cav.) de la Sota Córdoba: Dpto. Punilla. Pampa de Achala, pastizal, Arana s/n (3719 RCV). Dpto. Río Cuarto. Alpa Corral, bosque serrano, Arana s/n (2855 RCV).

Blechnum x pampasicum de la Sota \& Duran Córdoba: Dpto. Calamuchita. Sierra de Comechingones, Las Guindas, romerillal, Arana 610 (RCV, SI).

Blechnum cordatum (Desv.) Hieron.

Córdoba. Dpto. Punilla. Sierra Grande, falda este, ruta provincial 14, Copina, arroyo homónimo, 29-IV-1973, R. Subils 1761 (CORD).

Blechnum hastatum Kaulf.

Córdoba. Dpto. Calamuchita. Sierra de Comechingones, Alpa Corral, Cantero, Nuñez \& Gaich s/n (RIOC 2386).

Blechnum laevigatum Cav.

Córdoba: Dpto. Cruz del Eje. Sierra Grande, ruta provincial 28, km 804/805, río Guaste, 11-III-1986, R. Subils et al. 3912 bis (CORD).

Blechnum mochaenum G. Kunkel var. achalense (Hieron.) de la Sota

Córdoba. Dpto. Punilla. Las Ramadas cerca de Estancia San Miguel, Sierra de Achala, 14-III-1876, Hieronymus 478 (Isotipo CORD).
Blechnum penna-marina (Poir.) Kuhn

Córdoba: Dpto. Punilla. Sierra Grande, falda este, río Yuspe, 14-V1972, R. Subils \& Articó 1337 (CORD).

Blechnum squamipes (Hieron.) M. Kessler \& A.R. Sm.

Córdoba: Dpto. Punilla. Quebrada del Chorro, al este de Los Gigantes, Sierra de Achala, 3-XII-1878, Hieronymus s/n (Isotipo CORD).

\section{DRYOPTERIDACEAE}

Elaphoglossum gayanum (Fée) T. Moore

Córdoba: Dpto. Punilla. Cerro Blanco, Arana s/n (2012 RCV).

Elaphoglossum lorentzii (Hieron.) H. Christ

Córdoba: Dpto. Punilla. Pampa de Achala, pastizal, Arana s/n (3614 RCV).

Elaphoglossum piloselloides (C. Presl) T. Moore

Córdoba: Dpto. San Alberto. Pampa de Achala, 8-XI-1950, R. Capurro (BA 56227).

Polystichum montevidense (Spreng.) Rosenst. var. montevidense Córdoba: Dpto. Río Cuarto. Alpa Corral, Bosque serrano, en proximidades del Rio seco, Bianco 1026 (RIOC).

Polystichum montevidense (Spreng.) Rosenst var. squamulosa (Hieron.) Hieron.

Córdoba: Dpto. Calamuchita. Cerro Champaquí, Valetti s/n (3518 RCV).

\section{POLYPODIACEAE}

Melpomene peruviana (Desv.) A.R. Sm. \& R.C. Moran Córdoba: Dpto. Punilla. Sierras Grandes, más arriba de Copina, 24-XI-1932, Hosseus 52 y 53 (CORD).

Microgramma vacciniifolia (Langsd. \& Fisch.) Copel. Córdoba: Dpto. Sobremonte, en las proximidades de San Francisco del Chañar, 3955'S 6349'W, 573 ms.m., 21/II/2006, Giorgis, Cabido, Cingolani \& Gurvich 1044 (CORD).

Phlebodium areolatum (Willd.) J. Sm.

Córdoba: Dpto. Río Seco, Cerro Colorado, 2945'S 56³4'W, 18/ VI/2005, Morero 108 (CORD).

Pleopeltis minima (Bory) J. Prado \& R. Y. Hirai Córdoba: Dpto. Punilla. Sierras Chicas, IV-1904, Stuckert 15784 (CORD).

Pleopeltis pinnatifida Gillies ex Hook. \& Grev. Córdoba: Dpto. Calamuchita. Las Guindas, Arana s/n (2329 RCV).

Serpocaulon gilliesii (C. Chr.) A.R. Sm. Córdoba: Dpto. Punilla. Pampa de Achala, pastizal, Arana s/n (3702 RCV). 
Biogeografía y conservación de helechos de la Argentina central: ArANA, M. ET.AL.

APÉNDICE 3. Matrices de datos biogeográficos, la primera columna (ANCES) fue utilizada como grupo externo.

APPENDIX 3: Matrix of biogeographical data, the first column (ANCES) was used as outgroup.

A: presencia ausencia de los taxones por área de endemismo (ver acrónimos en el texto).

\begin{tabular}{|c|c|c|c|c|c|c|c|c|c|c|c|c|c|c|}
\hline $\begin{array}{c}\text { Especie/área de } \\
\text { endemismo }\end{array}$ & ANCES & SPA & CNA & $\mathrm{CHA}$ & MA & NA & PAM & PAT & $\mathrm{CHI}$ & SA & SB & TA & UR & VE \\
\hline $\begin{array}{l}\text { Adiantopsis } \\
\text { chlorophylla }\end{array}$ & 0 & 1 & 0 & 1 & 0 & 1 & 0 & 0 & 0 & 1 & 1 & 0 & 0 & 0 \\
\hline Adiantopsis tweediana & 0 & 1 & 0 & 1 & 0 & 0 & 1 & 0 & 0 & 0 & 1 & 0 & 1 & 0 \\
\hline $\begin{array}{l}\text { Adiantum } \\
\text { camptorachis }\end{array}$ & 0 & 1 & 0 & 0 & 0 & 1 & 0 & 0 & 1 & 1 & 0 & 0 & 0 & 0 \\
\hline Adiantum lorentzii & 0 & 1 & 0 & 1 & 0 & 1 & 0 & 0 & 0 & 1 & 1 & 0 & 0 & 0 \\
\hline $\begin{array}{l}\text { Adiantum } \\
\text { orbignyanum }\end{array}$ & 0 & 1 & 0 & 0 & 0 & 1 & 0 & 0 & 0 & 1 & 0 & 0 & 0 & 0 \\
\hline Adiantum raddianum & 0 & 1 & 0 & 1 & 0 & 1 & 1 & 0 & 0 & 1 & 1 & 1 & 0 & 1 \\
\hline $\begin{array}{l}\text { Adiantum } \\
\text { thalictroides f. bottini }\end{array}$ & 0 & 1 & 0 & 0 & 1 & 0 & 0 & 0 & 0 & 1 & 0 & 1 & 0 & 1 \\
\hline $\begin{array}{l}\text { Adiantum } \\
\text { thalictroides var. } \\
\text { thalictroides }\end{array}$ & 0 & 1 & 1 & 0 & 1 & 1 & 0 & 0 & 1 & 1 & 0 & 1 & 0 & 1 \\
\hline Anemia australis & 0 & 1 & 0 & 0 & 0 & 0 & 0 & 0 & 0 & 1 & 0 & 0 & 0 & 0 \\
\hline $\begin{array}{l}\text { Anemia tomentosa } \\
\text { var. anthriscifolia }\end{array}$ & 0 & 1 & 0 & 1 & 0 & 1 & 1 & 0 & 0 & 1 & 1 & 1 & 0 & 1 \\
\hline $\begin{array}{l}\text { Anemia tomentosa } \\
\text { var. tomentosa }\end{array}$ & 0 & 1 & 0 & 1 & 0 & 1 & 0 & 0 & 0 & 1 & 1 & 1 & 1 & 1 \\
\hline $\begin{array}{l}\text { Anogramma } \\
\text { chaerophylla }\end{array}$ & 0 & 1 & 1 & 1 & 0 & 1 & 1 & 0 & 0 & 1 & 0 & 1 & 1 & 1 \\
\hline Anogramma lorentzii & 0 & 1 & 0 & 0 & 0 & 0 & 1 & 0 & 0 & 1 & 1 & 0 & 1 & 0 \\
\hline $\begin{array}{l}\text { Argyrochosma nivea } \\
\text { var. flava }\end{array}$ & 0 & 1 & 0 & 0 & 0 & 1 & 0 & 0 & 0 & 1 & 1 & 0 & 0 & 0 \\
\hline $\begin{array}{l}\text { Argyrochosma nivea } \\
\text { var. nivea }\end{array}$ & 0 & 1 & 0 & 1 & 1 & 1 & 0 & 0 & 1 & 1 & 1 & 1 & 0 & 1 \\
\hline $\begin{array}{l}\text { Argyrochosma nivea } \\
\text { var. tenera }\end{array}$ & 0 & 1 & 0 & 0 & 1 & 1 & 0 & 0 & 0 & 1 & 1 & 1 & 0 & 1 \\
\hline Asplenium achalense & 0 & 1 & 0 & 0 & 0 & 0 & 0 & 0 & 0 & 1 & 0 & 0 & 0 & 0 \\
\hline $\begin{array}{l}\text { Asplenium } \\
\text { depauperatum }\end{array}$ & 0 & 1 & 0 & 1 & 0 & 1 & 0 & 0 & 0 & 1 & 1 & 0 & 0 & 0 \\
\hline Asplenium gilliesii & 0 & 1 & 0 & 0 & 0 & 1 & 0 & 0 & 0 & 1 & 0 & 1 & 0 & 1 \\
\hline Asplenium lilloanum & 0 & 1 & 0 & 0 & 0 & 0 & 0 & 0 & 0 & 1 & 0 & 0 & 0 & 0 \\
\hline Asplenium lorentzii & 0 & 1 & 0 & 0 & 0 & 1 & 0 & 0 & 0 & 1 & 0 & 0 & 0 & 0 \\
\hline Asplenium monanthes & 0 & 1 & 1 & 0 & 1 & 1 & 0 & 0 & 1 & 1 & 1 & 1 & 1 & 1 \\
\hline Asplenium resiliens & 0 & 1 & 1 & 0 & 0 & 1 & 0 & 0 & 0 & 1 & 1 & 1 & 1 & 1 \\
\hline Athyrium dombeyi & 0 & 1 & 0 & 0 & 0 & 1 & 0 & 0 & 0 & 1 & 1 & 1 & 0 & 1 \\
\hline Azolla filiculoides & 0 & 1 & 1 & 1 & 1 & 1 & 1 & 1 & 1 & 1 & 0 & 1 & 0 & 1 \\
\hline $\begin{array}{l}\text { Blechnum australe } \\
\text { ssp. auriculatum }\end{array}$ & 0 & 1 & 0 & 0 & 1 & 0 & 0 & 1 & 0 & 1 & 1 & 1 & 1 & 1 \\
\hline Blechnum cordatum & 0 & 1 & 0 & 0 & 0 & 0 & 0 & 1 & 1 & 0 & 1 & 1 & 0 & 1 \\
\hline Blechnum hastatum & 0 & 1 & 0 & 0 & 0 & 0 & 0 & 1 & 1 & 0 & 0 & 0 & 0 & 0 \\
\hline Blechnum laevigatum & 0 & 1 & 0 & 0 & 0 & 0 & 0 & 0 & 0 & 1 & 1 & 1 & 1 & 1 \\
\hline
\end{tabular}


Gayana Bot. 70(2), 2013

\begin{tabular}{|c|c|c|c|c|c|c|c|c|c|c|c|c|c|c|}
\hline $\begin{array}{l}\text { Blechnum mochaenum } \\
\text { ssp. achalense }\end{array}$ & 0 & 1 & 0 & 0 & 0 & 0 & 0 & 1 & 0 & 0 & 0 & 0 & 0 & 0 \\
\hline $\begin{array}{l}\text { Blechnum penna- } \\
\text { marina }\end{array}$ & 0 & 1 & 0 & 0 & 0 & 1 & 0 & 1 & 1 & 1 & 1 & 0 & 0 & 1 \\
\hline Blechnum squamipes & 0 & 1 & 0 & 0 & 0 & 0 & 0 & 0 & 0 & 1 & 0 & 0 & 0 & 0 \\
\hline $\begin{array}{l}\text { Blechnum } x \\
\text { pampasicum }\end{array}$ & 0 & 1 & 0 & 0 & 0 & 0 & 0 & 0 & 0 & 1 & 0 & 1 & 1 & 1 \\
\hline Botrychium australe & 0 & 1 & 0 & 0 & 0 & 0 & 0 & 0 & 0 & 1 & 0 & 1 & 0 & 1 \\
\hline Cassebeera triphylla & 0 & 1 & 0 & 1 & 0 & 0 & 0 & 0 & 0 & 1 & 1 & 1 & 1 & 1 \\
\hline Cheilanthes bonariensis & 0 & 1 & 1 & 0 & 1 & 1 & 0 & 0 & 1 & 1 & 0 & 0 & 0 & 0 \\
\hline Cheilanthes buchtienii & 0 & 1 & 0 & 0 & 1 & 1 & 0 & 0 & 0 & 1 & 0 & 1 & 0 & 1 \\
\hline Cheilanthes marginata & 0 & 1 & 1 & 0 & 0 & 1 & 0 & 0 & 0 & 1 & 0 & 0 & 0 & 0 \\
\hline Cheilanthes micropteris & 0 & 1 & 0 & 1 & 1 & 1 & 1 & 0 & 0 & 1 & 1 & 1 & 1 & 1 \\
\hline Cheilanthes myriophylla & 0 & 1 & 1 & 0 & 1 & 1 & 0 & 0 & 0 & 1 & 1 & 1 & 0 & 1 \\
\hline Cheilanthes obducta & 0 & 1 & 0 & 1 & 0 & 1 & 0 & 0 & 0 & 1 & 0 & 0 & 0 & 0 \\
\hline Cheilanthes pilosa & 0 & 1 & 0 & 0 & 0 & 1 & 0 & 0 & 0 & 1 & 0 & 0 & 0 & 0 \\
\hline Cheilanthes pruinata & 0 & 1 & 0 & 0 & 0 & 1 & 0 & 0 & 1 & 1 & 0 & 0 & 0 & 0 \\
\hline Cheilanthes squamosa & 0 & 1 & 0 & 0 & 0 & 1 & 0 & 0 & 0 & 1 & 0 & 1 & 0 & 1 \\
\hline Cystopteris diaphana & 0 & 1 & 0 & 0 & 0 & 1 & 0 & 1 & 1 & 1 & 0 & 0 & 0 & 0 \\
\hline Dennstaedtia globulifera & 0 & 1 & 0 & 0 & 0 & 1 & 0 & 0 & 0 & 1 & 0 & 1 & 1 & 1 \\
\hline Doryopteris concolor & 0 & 1 & 1 & 1 & 0 & 1 & 1 & 0 & 0 & 1 & 1 & 1 & 1 & 1 \\
\hline Doryopteris lorentzii & 0 & 1 & 0 & 1 & 0 & 1 & 0 & 0 & 0 & 1 & 1 & 0 & 1 & 0 \\
\hline Elaphoglossum gayanum & 0 & 1 & 1 & 0 & 0 & 1 & 0 & 0 & 1 & 1 & 1 & 1 & 0 & 1 \\
\hline Elaphoglossum lorentzii & 0 & 1 & 0 & 0 & 0 & 1 & 0 & 0 & 0 & 1 & 0 & 1 & 0 & 1 \\
\hline $\begin{array}{l}\text { Elaphoglossum } \\
\text { piloselloides }\end{array}$ & 0 & 1 & 0 & 0 & 0 & 1 & 0 & 0 & 1 & 1 & 1 & 0 & 0 & 0 \\
\hline Equisetum bogotense & 0 & 1 & 1 & 0 & 0 & 1 & 1 & 1 & 1 & 1 & 0 & 0 & 0 & 0 \\
\hline Equisetum giganteum & 0 & 1 & 1 & 1 & 1 & 1 & 1 & 1 & 1 & 1 & 1 & 1 & 1 & 1 \\
\hline $\begin{array}{l}\text { Hymenophyllum } \\
\text { tunbrigense var. } \\
\text { cordobense }\end{array}$ & 0 & 1 & 0 & 0 & 0 & 0 & 0 & 0 & 0 & 1 & 0 & 0 & 0 & 0 \\
\hline Hypolepis poeppigii & 0 & 1 & 1 & 0 & 0 & 1 & 0 & 0 & 0 & 1 & 1 & 0 & 0 & 0 \\
\hline Marsilea ancylopoda & 0 & 1 & 1 & 1 & 0 & 0 & 0 & 1 & 0 & 1 & 1 & 1 & 1 & 1 \\
\hline Melpomene peruviana & 0 & 1 & 0 & 0 & 0 & 1 & 0 & 0 & 0 & 1 & 1 & 1 & 0 & 1 \\
\hline $\begin{array}{l}\text { Microgramma } \\
\text { vacciniifolia }\end{array}$ & 0 & 1 & 0 & 1 & 0 & 0 & 0 & 0 & 0 & 0 & 1 & 0 & 1 & 0 \\
\hline Notholaena sulphurea & 0 & 1 & 1 & 0 & 0 & 1 & 0 & 0 & 0 & 1 & 0 & 0 & 0 & 0 \\
\hline $\begin{array}{l}\text { Ophioglossum } \\
\text { crotalophoroides ssp. } \\
\text { crotalophoroides }\end{array}$ & 0 & 1 & 1 & 1 & 1 & 1 & 1 & 1 & 1 & 1 & 0 & 1 & 1 & 1 \\
\hline $\begin{array}{l}\text { Ophioglossum } \\
\text { crotalophoroides var. } \\
\text { nanum }\end{array}$ & 0 & 1 & 0 & 1 & 0 & 0 & 0 & 0 & 0 & 0 & 0 & 0 & 0 & 0 \\
\hline Pellaea ternifolia & 0 & 1 & 1 & 1 & 1 & 1 & 1 & 0 & 1 & 1 & 1 & 1 & 0 & 1 \\
\hline Phlebodium areolatum & 0 & 1 & 1 & 0 & 0 & 0 & 0 & 0 & 0 & 1 & 1 & 0 & 0 & 0 \\
\hline Pilularia americana & 0 & 1 & 1 & 0 & 0 & 1 & 0 & 1 & 1 & 0 & 1 & 1 & 0 & 1 \\
\hline $\begin{array}{l}\text { Pityrogramma } \\
\text { calomelanos var. } \\
\text { austroamericana }\end{array}$ & 0 & 1 & 1 & 0 & 0 & 1 & 0 & 0 & 0 & 1 & 1 & 0 & 0 & 0 \\
\hline $\begin{array}{l}\text { Pityrogramma } \\
\text { calomelanos var. } \\
\text { calomelanos }\end{array}$ & 0 & 1 & 1 & 0 & 0 & 1 & 1 & 0 & 0 & 1 & 1 & 1 & 0 & 1 \\
\hline
\end{tabular}


Biogeografía y conservación de helechos de la Argentina central: ArANA, M. ET.AL.

\begin{tabular}{|c|c|c|c|c|c|c|c|c|c|c|c|c|c|c|}
\hline $\begin{array}{l}\text { Pityrogramma tartarea } \\
\text { var. aurata }\end{array}$ & 0 & 1 & 0 & 0 & 0 & 1 & 0 & 0 & 0 & 0 & 0 & 0 & 0 & 0 \\
\hline Pleopeltis minima & 0 & 1 & 0 & 1 & 0 & 1 & 0 & 0 & 0 & 1 & 1 & 1 & 1 & 1 \\
\hline Pleopeltis pinnatifida & 0 & 1 & 0 & 0 & 1 & 0 & 0 & 0 & 0 & 1 & 0 & 1 & 0 & 1 \\
\hline $\begin{array}{l}\text { Polystichum } \\
\text { montevidense var. } \\
\text { montevidense }\end{array}$ & 0 & 1 & 0 & 1 & 0 & 1 & 0 & 0 & 0 & 1 & 1 & 1 & 1 & 1 \\
\hline $\begin{array}{l}\text { Polystichum } \\
\text { montevidense var. } \\
\text { squamulosa }\end{array}$ & 0 & 1 & 0 & 0 & 0 & 1 & 0 & 0 & 0 & 1 & 0 & 0 & 0 & 0 \\
\hline Pteridium arachnoideum & 0 & 1 & 1 & 1 & 0 & 1 & 1 & 0 & 0 & 1 & 1 & 1 & 1 & 1 \\
\hline Serpocaulon gilliesii & 0 & 1 & 0 & 0 & 0 & 1 & 0 & 0 & 0 & 1 & 0 & 1 & 0 & 1 \\
\hline Thelypteris achalensis & 0 & 1 & 0 & 0 & 0 & 1 & 0 & 0 & 0 & 1 & 0 & 0 & 0 & 0 \\
\hline Thelypteris argentina & 0 & 1 & 0 & 0 & 0 & 1 & 0 & 1 & 1 & 1 & 0 & 1 & 1 & 1 \\
\hline Trismeria trifoliata & 0 & 1 & 1 & 1 & 1 & 1 & 1 & 0 & 0 & 1 & 1 & 1 & 0 & 1 \\
\hline Woodsia montevidensis & 0 & 1 & 0 & 0 & 1 & 1 & 0 & 1 & 0 & 1 & 1 & 1 & 1 & 1 \\
\hline
\end{tabular}

B: presencia ausencia de los taxones por centro de diversidad (ver acrónimos en el texto).

\begin{tabular}{|c|c|c|c|c|c|}
\hline TAXÓN/ CENTRO DE DIVERSIDAD & ANCES & Córdoba & CSNO & CSNE & CTS \\
\hline Adiantopsis chlorophylla & 0 & 1 & 1 & 1 & 0 \\
\hline Adiantopsis tweediana & 0 & 1 & 0 & 1 & 0 \\
\hline Adiantum lorentzii & 0 & 1 & 1 & 1 & 0 \\
\hline Adiantum orbignyanum & 0 & 1 & 1 & 0 & 0 \\
\hline Adiantum raddianum & 0 & 1 & 1 & 1 & 0 \\
\hline Adiantum thalictroides f. bottini & 0 & 1 & 1 & 0 & 0 \\
\hline Adiantum camptorachis & 0 & 1 & 1 & 0 & 0 \\
\hline Adiantum thalictroides var. thalictroides & 0 & 1 & 1 & 0 & 0 \\
\hline Anemia australis & 0 & 1 & 1 & 0 & 0 \\
\hline Anemia tomentosa var. anthriscifolia & 0 & 1 & 1 & 1 & 0 \\
\hline Anemia tomentosa var. tomentosa & 0 & 1 & 0 & 1 & 0 \\
\hline Anogramma chaerophylla & 0 & 1 & 1 & 1 & 0 \\
\hline Anogramma lorentzii & 0 & 1 & 1 & 1 & 0 \\
\hline Argyrochosma nivea var. flava & 0 & 1 & 1 & 0 & 0 \\
\hline Argyrochosma nivea var. nivea & 0 & 1 & 1 & 0 & 0 \\
\hline Argyrochosma nivea var. tenera & 0 & 1 & 1 & 0 & 0 \\
\hline Asplenium achalense & 0 & 1 & 1 & 0 & 0 \\
\hline Asplenium depauperatum & 0 & 1 & 1 & 1 & 0 \\
\hline Asplenium gilliesii & 0 & 1 & 1 & 0 & 0 \\
\hline Asplenium lilloanum & 0 & 1 & 1 & 0 & 0 \\
\hline Asplenium lorentzii & 0 & 1 & 1 & 0 & 0 \\
\hline Asplenium monanthes & 0 & 1 & 1 & 1 & 0 \\
\hline Asplenium resiliens & 0 & 1 & 1 & 0 & 0 \\
\hline Athyrium dombeyi & 0 & 1 & 1 & 0 & 0 \\
\hline Azolla filiculoides & 0 & 1 & 1 & 1 & 1 \\
\hline Blechnum australe subsp. auriculatum & 0 & 1 & 1 & 1 & 1 \\
\hline Blechnum cordatum & 0 & 1 & 0 & 0 & 1 \\
\hline Blechnum hastatum & 0 & 1 & 0 & 0 & 1 \\
\hline Blechnum laevigatum & 0 & 1 & 1 & 0 & 0 \\
\hline Blechnum mochaenum var. achalense & 0 & 1 & 0 & 0 & 1 \\
\hline Blechnum penna-marina & 0 & 1 & 1 & 0 & 1 \\
\hline
\end{tabular}


Gayana Bot. 70(2), 2013

\begin{tabular}{|c|c|c|c|c|c|}
\hline Blechnum squamipes & 0 & 1 & 1 & 0 & 0 \\
\hline Blechnum x pampasicum & 0 & 0 & 0 & 1 & 0 \\
\hline Botrychium australe & 0 & 1 & 1 & 0 & 0 \\
\hline Cassebeera triphylla & 0 & 1 & 1 & 1 & 0 \\
\hline Cheilanthes bonariensis & 0 & 1 & 1 & 0 & 0 \\
\hline Cheilanthes buchtienii & 0 & 1 & 1 & 0 & 0 \\
\hline Cheilanthes marginata & 0 & 1 & 1 & 0 & 0 \\
\hline Cheilanthes micropteris & 0 & 1 & 1 & 1 & 0 \\
\hline Cheilanthes myriophylla & 0 & 1 & 1 & 1 & 0 \\
\hline Cheilanthes obducta & 0 & 1 & 1 & 0 & 0 \\
\hline Cheilanthes pilosa & 0 & 1 & 1 & 0 & 0 \\
\hline Cheilanthes pruinata & 0 & 1 & 1 & 0 & 0 \\
\hline Cheilanthes squamosa & 0 & 1 & 1 & 0 & 0 \\
\hline Cystopteris diaphana & 0 & 1 & 1 & 0 & 0 \\
\hline Dennstaedtia globulifera & 0 & 1 & 1 & 1 & 0 \\
\hline Doryopteris concolor & 0 & 1 & 1 & 1 & 0 \\
\hline Doryopteris lorentzii & 0 & 1 & 1 & 1 & 0 \\
\hline Elaphoglossum gayanum & 0 & 1 & 1 & 0 & 0 \\
\hline Elaphoglossum lorentzii & 0 & 1 & 1 & 0 & 0 \\
\hline Elaphoglossum piloselloides & 0 & 1 & 1 & 0 & 0 \\
\hline Equisetum bogotense & 0 & 1 & 1 & 0 & 1 \\
\hline Equisetum giganteum & 0 & 1 & 1 & 1 & 1 \\
\hline Hymenophyllum tunbrigense var. cordobense & 0 & 1 & 1 & 0 & 0 \\
\hline Hypolepis poeppigii & 0 & 1 & 1 & 0 & 0 \\
\hline Marsilea ancylopoda & 0 & 1 & 1 & 1 & 1 \\
\hline Melpomene peruviana & 0 & 1 & 1 & 0 & 0 \\
\hline Microgramma vacciniifolia & 0 & 1 & 0 & 1 & 0 \\
\hline Notholaena sulphurea & 0 & 1 & 1 & 0 & 0 \\
\hline $\begin{array}{l}\text { Ophioglossum crotalophoroides subsp. } \\
\text { crotalophoroides }\end{array}$ & 0 & 1 & 1 & 1 & 1 \\
\hline Ophioglossum crotalophoroides var. nanum & 0 & 1 & 0 & 0 & 0 \\
\hline Pellaea ternifolia & 0 & 1 & 1 & 1 & 0 \\
\hline Phlebodium areolatum & 0 & 1 & 1 & 1 & 0 \\
\hline Pilularia americana & 0 & 1 & 0 & 1 & 1 \\
\hline Pityrogramma calomelanos var. austroamericana & 0 & 1 & 1 & 0 & 0 \\
\hline Pityrogramma calomelanos var. calomelanos & 0 & 1 & 1 & 1 & 0 \\
\hline Pityrogramma tartarea var. aurata & 0 & 1 & 0 & 0 & 0 \\
\hline Pleopeltis minima & 0 & 1 & 1 & 1 & 0 \\
\hline Pleopeltis pinnatifida & 0 & 1 & 1 & 0 & 0 \\
\hline Polystichum montevidense var. montevidense & 0 & 1 & 1 & 1 & 0 \\
\hline Polystichum montevidense var. squamulosa & 0 & 1 & 1 & 0 & 1 \\
\hline Pteridium arachnoideum & 0 & 1 & 1 & 1 & 0 \\
\hline Serpocaulon gilliesii & 0 & 1 & 1 & 0 & 0 \\
\hline Thelypteris achalensis & 0 & 1 & 1 & 0 & 0 \\
\hline Thelypteris argentina & 0 & 1 & 1 & 0 & 0 \\
\hline Trismeria trifoliata & 0 & 1 & 1 & 1 & 0 \\
\hline Woodsia montevidensis & 0 & 1 & 1 & 0 & 1 \\
\hline
\end{tabular}

Recibido: 31.08 .11

Aceptado: 23.04.13 